Discussion Paper No. 823

\title{
MAXIMAL DOMAIN \\ FOR STRATEGY-PROOF \\ PROBABILISTIC RULES \\ IN ECONOMIES \\ WITH ONE PUBLIC GOOD
}

\author{
Shuhei Morimoto
}

November 2011

The Institute of Social and Economic Research Osaka University

6-1 Mihogaoka, Ibaraki, Osaka 567-0047, Japan 


\title{
Maximal Domain for Strategy-proof Probabilistic Rules in Economies with One Public Good*
}

\author{
Shuhei Morimoto \\ Institute of Social and Economic Research, Osaka University \\ 6-1, Mihogaoka, Ibaraki, 567-0047, Japan \\ E-mail: morimoto@iser.osaka-u.ac.jp
}

November 17, 2011

\begin{abstract}
We consider the problem of choosing a level of a public good on an interval of the real line among a group of agents. A probabilistic rule chooses a probability distribution over the interval for each preference profile. We investigate strategy-proof probabilistic rules in the case where distributions are compared based on stochastic dominance relations. First, on a "minimally rich domain", we characterize the so-called probabilistic generalized median rules (Ehlers et al., 2002, Journal of Economic Theory 105: 408-434) by means of stochastic-dominance (sd) strategy-proofness and ontoness. Next, we study how much we can enlarge a domain to allow for the existence of sd-strategy-proof probabilistic rules that satisfy ontoness and the no-vetoer condition. We establish that the domain of "convex" preferences is the unique maximal domain including a minimally rich domain for these properties.
\end{abstract}

Keywords: public good, probabilistic rule, stochastic dominance relation, strategy-proofness, minimally rich domain, maximal domain

JEL Classification Numbers: D71

\footnotetext{
*I am very grateful to William Thomson for his many detailed and helpful comments. I also acknowledge the useful comments by participants at the 2011 Autumn Annual Meeting of the Japanese Economic Association in University of Tsukuba. This paper was partially written while I was visiting the Department of Economics at the University of Rochester. I appreciate the good research environment of the department, and acknowledge financial support from the Research Fellowships for Young Scientists of the Japan Society for the Promotion of Science. All remaining errors are my own.
} 


\section{Introduction}

We consider the problem of choosing a level of a public good on an interval of the real line among a group of agents. Each agent has ordinal preferences over the chosen level of the public good. We examine this problem in a probabilistic framework to achieve a "fair" solution. Consider a situation where a group of agents try to choose one of two public projects, $a$ and $b$. Assume that half of them prefer $a$ to $b$, while the other half prefer $b$ to $a$. In this case, one of the fair solutions to this problem would be to use a lottery between the two projects. ${ }^{1}$ A probabilistic rule, or simply a rule, chooses a probability distribution over the interval for each preference profile. ${ }^{2}$

Since preferences are defined over deterministic alternatives and the probabilistic rule selects the probability distribution, we need to explain how agents evaluate distributions. In this paper, we assume that each agent compares probability distributions based on (first order) stochastic dominance relation derived from his ordinal preference. That is, for each preference of the agent, and each pair of probability distributions, say $f$ and $g, f$ stochastically dominates $g$ according to his preference if to each upper contour set of the preference, $f$ assigns a probability at least as high as the probability assigned under $g$. This is equivalent to assuming that for each von Neumann-Morgenstern utility function that represents the agent's ordinal preference, the expected utility under the distribution $f$ is at least as high as the expected utility under $g$.

Preferences are usually private information. Agents may strategically misrepresent their preferences to obtain the outcome distributions they prefer. As a result, the chosen outcome may not be socially desirable relative to the agents' true preferences. The property called stochastic-dominance (sd) strategy-proofness is often imposed for a probabilistic rule to give agents the incentive to represent their preferences truthfully. It requires that for each agent, truth-telling stochastically dominates lying.

It is well-known that there is no sd-strategy-proof and ex-post efficient probabilistic rule, defined on the unrestricted domain, other than the random dictatorship rules when there are at least three alternatives (Gibbard, 1977). However, if the domain is restricted to the class of "single-peaked" preferences, Ehlers et al. (2002) show that there are sd-strategy-proof probabilistic rules other than random dictatorships. ${ }^{3}$ A preference is single-peaked if there is a unique best alternative, called the peak, such that its welfare monotonically decreases around the peak. On the single-peaked domain, they also characterize the class of the so-called probabilistic generalized median rules by means of sd-strategy-proofness and unanimity. ${ }^{4}$ Thus,

\footnotetext{
${ }^{1}$ Ehlers and Klaus (2001) and Ehlers et al. (2002) also consider a probabilistic approach to improve "a priori fairness".

${ }^{2}$ Note that the probabilistic rules studied in this paper rely on only ordinal preferences of agents. This approach is usual in the context of voting problems with lotteries. For example, see Gibbard (1977) and Barberà (1979a,b).

${ }^{3} \mathrm{On}$ the other hand, when the set of alternatives is a convex subset of multi-dimensional Euclidean space, Dutta et al. (2002) show that, on the domain of all strictly convex and single-peaked preferences, a rule is sd-strategy-proof and unanimous if and only if it is a random dictatorship.

${ }^{4}$ Ehlers and Klaus (2001) examine the one-dimensional voting problem from the perspective of
} 
they extend the results of Moulin $(1980)^{5}$ to the probabilistic model. In this paper, following the results of Ehlers et al. (2002), we examine the following two questions on the domain conditions of sd-strategy-proof probabilistic rules.

The first question is how much we can restrict the domain on which the probabilistic generalized median rules are the unique class of sd-strategy-proof and onto rules. If a rule is sd-strategy-proof on a domain, then it is also sd-strategy-proof on any subdomain. Thus, the smaller the domain, the weaker the requirement of sd-strategy-proofness. In this paper, a "minimally rich domain" is defined as a small subset of the single-peaked domain satisfying the following two conditions: (1) for each alternative, there is "only one" preference whose peak is equal to the alternative, and (2) given three distinct alternatives, say $x, y$, and $z$ with $x<z<y$, there is a finite sequence of preferences whose peaks monotonically increase from $x$ to $z$ (respectively, decrease from $y$ to $z$ ) such that for each preference in the sequence, the previous peak point is preferred to $y$ (respectively, $x$ ). The "symmetric singlepeaked domain" ${ }^{6}$ is an example of the minimally rich domain. We establish that the probabilistic generalized median rules are the unique class of sd-strategy-proof and onto rules on a minimally rich domain.

The second question is how much we can enlarge a domain to allow for the existence of sd-strategy-proof and onto probabilistic rules. Note that the random dictatorship rules are sd-strategy-proof and onto on the universal domain. Thus, to rule out such trivial rules, we additionally impose the no-vetoer condition. Agent $i$ is a vetoer for a rule if the alternative that is best for all agents other than $i$, and worst for $i$, is never chosen with probability one. The no-vetoer condition requires that a rule should have no vetoer. We establish that the domain of "convex" preferences is the unique maximal domain including a minimally rich domain for the existence of probabilistic rules that satisfy sd-strategy-proofness, ontoness, and the no-vetoer condition.

Our results are closely related to those of Berga and Serizawa (2000). For the deterministic case, they characterize the class of generalized median rules by means of strategy-proofness and ontoness on a BS-minimally rich domain, ${ }^{7}$ and show that the convex domain is the unique maximal domain including a BS-minimally rich domain for strategy-proofness, ontoness, and the BS-no-vetoer condition. ${ }^{8}$ Under sd-strategy-proofness, the BS-no-vetoer condition implies the no-vetoer condition.

a solidarity property on the single-peaked domain.

${ }^{5}$ See also Ching (1997) for a detailed analysis of the deterministic model.

${ }^{6} \mathrm{~A}$ preference is symmetric single-peaked if there is a unique peak, and its utility representation is symmetric around the peak.

${ }^{7}$ Berga-Serizawa (BS)-minimally rich domain is a small subset of the single-peaked domain satisfying the following two conditions: (a) for each alternative, there is only one preference whose peak is equal to the alternative, and (b) given two distinct alternatives, say $x$ and $y$, there is a preference whose peak is between $x$ and $y$ such that $x$ is preferred to $y$. Condition (a) is the same as condition (1) of our minimally rich domain. Condition (b) is weaker than condition (2) proposed in this paper.

${ }^{8}$ The BS-no-vetoer condition requires that for each agent, each preference of the agent, and each alternative, there is a preference profile of the other agents at which the alternative is chosen with probability one. 
Thus, our results can be interpreted as an extension of those of Berga and Serizawa (2000) to the probabilistic model.

For the deterministic model, several papers examine the maximal domain for the existence of strategy-proof rules. Many authors such as Barberà et al. (1991, 1999), Serizawa (1995), and Berga (2002) investigate the maximal domain on which the generalized median rules are strategy-proof. Their analyses exclude rules other than the generalized median rules. On the other hand, Berga and Serizawa (2000) do not restrict the rules a priori, but obtain the maximal domain by only imposing properties on rules. To the best of our knowledge, our paper is the first to examine the maximal domain of sd-strategy-proof probabilistic rules. Further, we do not restrict the rules a priori, and establish the maximal domain result for properties on rules. Recently, Hatsumi et al. (2011) examine the maximal domain for the deterministic model with multiple public goods. They establish that the domain of separable preferences is a maximal domain for strategy-proofness and the $B S$ no-vetoer condition. For the allotment problem, Mizobuchi and Serizawa (2006) investigate the two questions that are parallel to Berga and Serizawa (2000) and our paper.

As we mentioned above, since the symmetric single-peaked domain is minimally rich, we also obtain the counterpart of the result of Border and Jordan $(1983)^{9}$ for the probabilistic case as a corollary of our result.

This paper is organized as follows. In Section 2, we present the model and definitions. Section 3 explains the main results. Section 4 provides the concluding remarks. Section 5 is devoted to the proofs of the results in Section 3.

\section{The model and definitions}

Let $N \equiv\{1, \ldots, n\}$ be a set of agents. Assume that $2 \leq n<\infty$. The set of alternatives is a closed interval $Z \equiv[\underline{z}, \bar{z}]$ on the real line $\mathbb{R}{ }^{10}$ A preference is a complete and transitive binary relation $R_{i}$ on $Z$. Let $P_{i}$ be the strict preference relation associated with $R_{i}$, and $I_{i}$ the indifference relation. Given a preference $R_{i}$ and $z \in Z$, the upper contour set of $\boldsymbol{R}_{\boldsymbol{i}}$ at $\boldsymbol{z}$ is the set $\boldsymbol{U}\left(\boldsymbol{R}_{\boldsymbol{i}}, \boldsymbol{z}\right) \equiv\left\{y \in Z: y R_{i}\right.$ $z\}$, and the lower contour set of $\boldsymbol{R}_{\boldsymbol{i}}$ at $\boldsymbol{z}$ is the set $\boldsymbol{L}\left(\boldsymbol{R}_{\boldsymbol{i}}, \boldsymbol{z}\right) \equiv\left\{y \in Z: z R_{i} y\right\}$. A preference $R_{i}$ is continuous if for each $z \in Z, U\left(R_{i}, z\right)$ and $L\left(R_{i}, z\right)$ are closed. Given a preference $R_{i}$, let $\boldsymbol{p}\left(\boldsymbol{R}_{\boldsymbol{i}}\right) \equiv\left\{x \in Z\right.$ : for each $\left.y \in Z, x R_{i} y\right\}$ be the set of the most preferred alternatives according to $R_{i}$. We call $p\left(R_{i}\right)$ the peak of $R_{i}$. Let $\mathcal{P}$ denote the set of continuous preference relations on $Z$. A domain is a subset $\mathcal{R}$ of $\mathcal{P}$. A preference profile is a list $R \equiv\left(R_{1}, \ldots, R_{n}\right) \in \mathcal{R}^{N}$. Let $R_{-i} \equiv\left(R_{j}\right)_{j \in N \backslash\{i\}}$ be a list of preferences for all agents except for agent $i$. We write the profile $\left(R_{1}, \ldots, R_{i-1}, \bar{R}_{i}, R_{i+1}, \ldots, R_{n}\right)$ as $\left(\bar{R}_{i}, R_{-i}\right)$.

A preference $R_{i}$ on $Z$ is convex if for each $z \in Z$, the set $U\left(R_{i}, z\right)$ is convex.

\footnotetext{
${ }^{9}$ For the deterministic case, they establish that on the symmetric single-peaked domain, the generalized median rules are the unique class of strategy-proof and onto rules.

${ }^{10}$ Given $\left.a, b \in \mathbb{R},[a, b],\right] a, b[,[a, b[$, and $] a, b]$ denote the closed, open, right half open, and left half open intervals with endpoints $a$ and $b$, respectively.
} 
Let $\mathcal{R}^{\text {vex }} \subset \mathcal{P}$ be the domain of convex preferences. A preference $R_{i}$ on $Z$ is singlepeaked if $p\left(R_{i}\right)$ is a singleton, and for each pair $z, z^{\prime} \in Z$, whenever $z<z^{\prime} \leq p\left(R_{i}\right)$ or $p\left(R_{i}\right) \leq z^{\prime}<z$, then $z^{\prime} P_{i} z$. Let $\mathcal{R}^{\sin } \subset \mathcal{P}$ be the domain of single-peaked preferences. A preference $R_{i}$ on $Z$ is symmetric single-peaked if $p\left(R_{i}\right)$ is a singleton, and for each pair $z, z^{\prime} \in Z,\left(z-p\left(R_{i}\right)\right)^{2}>\left(z^{\prime}-p\left(R_{i}\right)\right)^{2}$ if and only if $z^{\prime} P_{i} z$. Let $\mathcal{R}^{\text {sym }} \subset \mathcal{P}$ be the domain of symmetric single-peaked preferences. Note that $\mathcal{R}^{\text {sym }} \subsetneq \mathcal{R}^{\text {sin }} \subsetneq \mathcal{R}^{\text {vex }}$.

Let $\Delta(Z)$ be the set of probability distributions over $Z{ }^{11}$ A probabilistic rule $f$ is a function from $\mathcal{R}^{N}$ to $\Delta(Z)$. Throughout the paper, we simply refer to a probabilistic rule as rule. Given a subset $X$ of $Z, f(R)(X)$ is the probability that the chosen alternative belongs to $X$. We will also need to refer to rules that select degenerate distributions, that is, probability distributions that put weight one on a single alternative. A deterministic rule $F$ is a function from $\mathcal{R}^{N}$ to $Z$.

Since preferences are defined over deterministic alternatives and the rule selects probability distribution, we need to explain how agents evaluate distributions. Let $R_{i} \in \mathcal{R}$. The (first order) stochastic dominance relation (sd) associated with $R_{i}$ is defined as follows. For each pair $Q, Q^{\prime} \in \Delta(Z), \boldsymbol{Q}$ stochastically dominates $\boldsymbol{Q}^{\prime}$ at $\boldsymbol{R}_{\boldsymbol{i}}$ if for each $z \in Z, Q\left(U\left(R_{i}, z\right)\right) \geq Q^{\prime}\left(U\left(R_{i}, z\right)\right)$, We write this as $\boldsymbol{Q} \boldsymbol{R}_{\boldsymbol{i}}^{\boldsymbol{s} \boldsymbol{d}} \boldsymbol{Q}^{\prime}$. Also, $\boldsymbol{Q}$ strictly stochastically dominates $\boldsymbol{Q}^{\prime}$ at $\boldsymbol{R}_{\boldsymbol{i}}$ if $Q R_{i}^{s d} Q^{\prime}$, and for some $y \in Z, Q\left(U\left(R_{i}, y\right)\right)>Q^{\prime}\left(U\left(R_{i}, y\right)\right)$. We write this as $\boldsymbol{Q} \boldsymbol{P}_{\boldsymbol{i}}^{\boldsymbol{s d}} \boldsymbol{Q}^{\prime}$.

Next, we introduce the axioms. Let $f$ be a rule on $\mathcal{R}^{N}$. Following the terminology given by Thomson (2010), we use the prefix "sd" in naming the axioms based on stochastic dominance relations. The next two axioms are related to incentive compatibility. First, for each agent, truth-telling stochastically dominates lying.

Sd-strategy-proofness: For each $R \in \mathcal{R}^{N}$, each $i \in N$, and each $\hat{R}_{i} \in \mathcal{R}$, $f(R) R_{i}^{s d} f\left(\hat{R}_{i}, R_{-i}\right)$.

Second, by misrepresenting his preferences, no agent can ever obtain a distribution that strictly stochastically dominates truth-telling.

Weak sd-strategy-proofness: For each $R \in \mathcal{R}^{N}$, and each $i \in N$, there is no $\hat{R}_{i} \in \mathcal{R}$ such that $f\left(\hat{R}_{i}, R_{-i}\right) P_{i}^{s d} f(R)$.

The next three axioms are related to efficiency. A distribution $Q \in \Delta(Z)$ is sd-efficient for $\boldsymbol{R} \in \mathcal{R}^{N}$ if there is no $Q^{\prime} \in \Delta(Z)$ such that for each $i \in N$, $Q^{\prime} R_{i}^{s d} Q$, and for some $j \in N, Q^{\prime} P_{j}^{s d} Q$.

Sd-efficiency: ${ }^{12}$ For each $R \in \mathcal{R}^{N}, f(R)$ is sd-efficient for $R$.

Second, if there are alternatives that all agents agree as the best, then these alternatives should be chosen with probability 1 .

Unanimity: For each $R \in \mathcal{R}^{N}$ such that $\bigcap_{i \in N} p\left(R_{i}\right) \neq \emptyset, f(R)\left(\bigcap_{i \in N} p\left(R_{i}\right)\right)=1$.

\footnotetext{
${ }^{11}$ We consider probability distributions defined on the Borel $\sigma$-algebra $\mathcal{L}$ on $Z$. See the Appendix in Ehlers et al. (2002) for the formal definition. Throughout the paper, we assume that each subset $X$ of $Z$ belongs to $\mathcal{L}$. Also, we often refer to several definitions and results found in Ehlers et al. (2002).

${ }^{12}$ This notion is also referred to as ordinal efficiency.
} 
Third, for each alternative, there is a preference profile at which the alternative is chosen with probability 1 .

Ontoness: For each $z \in Z$, there is $R \in \mathcal{R}^{N}$ such that $f(R)(\{z\})=1$.

Note that sd-efficiency implies unanimity, which implies ontoness.

The next three properties pertain to how a rule distributes power across agents. Agent $i \in N$ is a dictator for a rule if the outcome is always chosen from this agent's best alternatives. Non-dictatorship requires that a rule should have no dictator.

Non-dictatorship: For each $i \in N$, there is $R \in \mathcal{R}^{N}$ such that $f(R)\left(p\left(R_{i}\right)\right) \neq 1$.

Agent $i \in N$ is a vetoer for a rule if the alternative that is best for all agents other than $i$, and worst for $i$, is never chosen with probability 1 . The no-vetoer condition requires that a rule should have no vetoer.

No-vetoer condition: For each $i \in N$, each $x \in Z$, and each $R \in \mathcal{R}^{N}$, if for each $j \in N \backslash\{i\},\{x\}=p\left(R_{j}\right)$, and for each $z \in Z, z R_{i} x$, then $f(R)(\{x\})=1$.

Note that the no-vetoer condition implies non-dictatorship. The following related condition is imposed in Berga and Serizawa (2000).

BS-no-vetoer condition: For each $i \in N$, each $x \in Z$, and each $R_{i} \in \mathcal{R}$, there is $R_{-i} \in \mathcal{R}^{N \backslash\{i\}}$ such that $f(R)(\{x\})=1$.

Note that under sd-strategy-proofness, the BS-no-vetoer condition implies the no-vetoer condition. ${ }^{13}$

The next property says that a rule only depends on the profile of peaks.

Peak-onlyness: For each pair $R, \hat{R} \in \mathcal{R}^{N}$, if for each $i \in N, p\left(R_{i}\right)=p\left(\hat{R}_{i}\right)$, then $f(R)=f(\hat{R})$.

Next is the class of rules introduced by Ehlers et al. (2002). It is central to our paper as well. The rules are described in the following way. Let $D \equiv\left(D_{S}\right)_{S \in 2^{N}}$ be a collection of probability distributions such that (i) $D_{\emptyset}(\{\bar{z}\})=1$, (ii) $D_{N}(\{\underline{z}\})=1$, and (iii) for each $S, T \in 2^{N}$, and each $x \in Z, D_{S \cup T}([\underline{z}, x])-D_{S}([\underline{z}, x[) \geq 0$. A unique rule is associated to each collection. We therefore refer to the collection as the "signature" of the rule. Let $\Delta$ be the set of signatures. As we will see, these distributions are the choices made by the rule for profiles of extremists. A step in the proof consists in identifying the collection. We refer to this step as a calibration step. $^{14}$

\footnotetext{
${ }^{13}$ To see this, let $f$ be a rule satisfying sd-strategy-proofness and BS-no-vetoer condition on $\mathcal{R}^{N}$. Let $i \in N, x \in Z$, and $R \in \mathcal{R}^{N}$ be such that for each $j \in N \backslash\{i\},\{x\}=p\left(R_{j}\right)$, and for each $z \in Z, z R_{i} x$. Then, by BS-no-vetoer condition, there is $\hat{R}_{-i} \in \mathcal{R}^{N \backslash\{i\}}$ such that $f\left(R_{i}, \hat{R}_{-i}\right)(\{x\})=1$. Without loss of generality, let $i=1$. By sd-strategyproofness, $f\left(R_{1}, R_{2}, \hat{R}_{-1,2}\right)(\{x\})=f\left(R_{1}, R_{2}, \hat{R}_{-1,2}\right)\left(U\left(R_{2}, x\right)\right) \geq f\left(R_{1}, \hat{R}_{-1}\right)\left(U\left(R_{2}, x\right)\right)=$ $f\left(R_{1}, \hat{R}_{-1}\right)(\{x\})=1$. Also, by sd-strategy-proofness, $f\left(R_{1}, R_{2,3}, \hat{R}_{-1,2,3}\right)(\{x\})=$ $f\left(R_{1}, R_{2,3}, \hat{R}_{-1,2,3}\right)\left(U\left(R_{3}, x\right)\right) \geq f\left(R_{1}, R_{2}, \hat{R}_{-1,2}\right)\left(U\left(R_{3}, x\right)\right)=f\left(R_{1}, R_{2}, \hat{R}_{-1,2}\right)(\{x\})=1$. Repeating this argument for agents $j=4, \ldots, n$, we have $f(R)(\{x\})=1$.

${ }^{14}$ This terminology was introduced by Thomson (1999).
} 
Given $R \in \mathcal{R}^{N}$, let $\bar{n}(R)$ be the number of different peaks at $R,{ }^{15}$ and for each $\ell \in\{1, \ldots, \bar{n}\}$, let $p^{\ell}(R)$ be the $\ell$-th smallest peak at $R$, and let $S_{\ell} \equiv\{i \in N$ : $\left.p\left(R_{i}\right) \leq p^{\ell}(R)\right\}, S_{0} \equiv \emptyset, p^{0}(R) \equiv \underline{z}$, and $p^{\bar{n}+1}(R) \equiv \bar{z}$.

We now define the class of rules.

Probabilistic generalized median rule with signature $D \in \Delta, g^{D}:^{16}$ For each $R \in \mathcal{R}^{N}$, and each $X \subset Z$,

$$
\begin{aligned}
g m^{D}(R)(X)=\sum_{\ell=0}^{\bar{n}} D_{S_{\ell}}(X \cap & ] p^{\ell}(R), p^{\ell+1}(R)[) \\
& +\sum_{\ell=1}^{\bar{n}} 1_{X}\left(p^{\ell}(R)\right)\left(D_{S_{\ell}}\left(\left[\underline{z}, p^{\ell}(R)\right]\right)-D_{S_{\ell-1}}\left(\left[\underline{z}, p^{\ell}(R)[)\right),\right.\right.
\end{aligned}
$$

where $1_{X}$ is the indicator function $1_{X}: Z \rightarrow\{0,1\}$ such that if $z \in X, 1_{X}(z)=1$, and otherwise, $1_{X}(z)=0 .{ }^{17}$

The following is a description of the probabilistic generalized median rule with signature $D \in \Delta, g m^{D}$. The distribution chosen by $\mathrm{gm}^{D}$ coincides with the distribution $D_{\emptyset}$ on the interval $\left[\underline{z}, p^{1}(R)\left[\right.\right.$, with $D_{N}$ on the interval $\left.] p^{\bar{n}}(R), \bar{z}\right]$, and for each $\ell \in\{1, \ldots, \bar{n}-1\}$, with $D_{S_{\ell}}$ on the interval $] p^{\ell}(R), p^{\ell+1}(R)[$. Further, for each $\ell \in\{1, \ldots, \bar{n}\}$, the probability assigned to the $\ell$-th peak, $\operatorname{gm}^{D}(R)\left(\left\{p^{\ell}(R)\right\}\right)$, is equal to $D_{S_{\ell}}\left(\left[\underline{z}, p^{\ell}(R)\right]\right)-D_{S_{\ell-1}}\left(\left[\underline{z}, p^{\ell}(R)[)\right.\right.$.

\section{The results}

\subsection{Characterization}

Ehlers et al. (2002) characterize the class of sd-strategy-proof and onto rules on the domain of single-peaked preferences.

Theorem (Ehlers et al., 2002). A rule defined on the domain of single-peaked preferences is sd-strategy-proof and onto if and only if it is a probabilistic generalized median rule. ${ }^{18}$

This section shows that their results still hold on more restricted domains. We establish it on a "minimally rich" domain defined as follows. For each pair $x, y \in Z$ with $x \neq y$, let $\mathcal{R}(y, x) \equiv\left\{R_{i} \in \mathcal{R}: x P_{i} y\right.$ and $\left.\min \{x, y\}<p\left(R_{i}\right)<\max \{x, y\}\right\}$.

\footnotetext{
${ }^{15}$ We simply use $\bar{n}$ when we can omit $R$ as an argument without confusion.

${ }^{16}$ We follow the terminology given by Thomson (2010). In Ehlers et al. (2002), this class of rules is referred to as "fixed-probabilistic-ballots rules".

${ }^{17}$ Since a probabilistic generalized median rule $g m^{D}$ is peak-only, we can denote $g m^{D}(R)$ by $g m^{D}\left(p\left(R_{i}\right)_{i \in N}\right)$ as a function of the profile of peaks.

${ }^{18}$ More precisely, unanimity is imposed in their paper instead of ontoness. Note that on the single-peaked preference domain, under sd-strategy-proofness, unanimity coincides with ontoness (See Fact 5 in the Appendix). They also characterize the class of sd-strategy-proof and peakonly rules on the single-peaked domain. This class of rules is slightly larger than the class of probabilistic generalized median rules.
} 
Definition 1. A domain $\mathcal{R} \subset \mathcal{P}$ is minimally rich if

(i) $\mathcal{R}$ is a subclass of the domain $\mathcal{R}^{\text {sin }}$ of single-peaked preferences,

(ii) for each $z \in Z$, there is a unique preference $R^{0} \in \mathcal{R}$ such that $p\left(R^{0}\right)=z$, and

(iii) for each triple $x, y, z \in Z$ with $x \neq y$ and $\min \{x, y\}<z<\max \{x, y\}$, there exist a finite $K \in \mathbb{N}$ and a sequence $\left\{R_{0}^{(k)}\right\}_{k=1}^{K}$ of preferences in $\mathcal{R}$ such that ${ }^{19}$

(iii-a) $R_{0}^{(1)} \in \mathcal{R}(y, x)$,

(iii-b) for each $k \in\{2, \ldots, K\}, R_{0}^{(k)} \in \mathcal{R}\left(y, p\left(R_{0}^{(k-1)}\right)\right)$, and

(iii-c) $\min \left\{p\left(R_{0}^{(K-1)}\right), p\left(R_{0}^{(K)}\right)\right\} \leq z \leq \max \left\{p\left(R_{0}^{(K-1)}\right), p\left(R_{0}^{(K)}\right)\right\}$, and $z \neq p\left(R_{0}^{(K-1)}\right)$.

Conditions (i) and (ii) say that, for each alternative, there is only one singlepeaked preference whose peak is equal to the alternative. Condition (iii) says that, given three distinct alternatives, say $x, y$, and $z$ with $\min \{x, y\}<z<\max \{x, y\}$, there is a finite sequence $\left\{R_{0}^{(k)}\right\}_{k=1}^{K}$ of preferences satisfying the following three conditions: (iii-a) the peak of $R_{0}^{(1)}$ is between $x$ and $y$, and $x$ is preferred to $y$ under $R_{0}^{(1)}$, (iii-b) for each $k \in\{2, \ldots, K\}$, the peak of $R_{0}^{(k)}$ is between $p\left(R_{0}^{(k-1)}\right)$ and $y$, and $p\left(R_{0}^{(k-1)}\right)$ is preferred to $y$ under $R_{0}^{(k)}$, and (iii-c) $z$ is between $p\left(R_{0}^{(K-1)}\right)$ and $p\left(R_{0}^{(K)}\right)$.

The following related condition is imposed in Berga and Serizawa (2000).

Definition 2. A domain $\mathcal{R} \subset \mathcal{P}$ is $B S$-minimally rich if (BS-i) $\mathcal{R}$ is a subclass of the domain $\mathcal{R}^{\text {sin }}$ of single-peaked preferences,

(BS-ii) for each $z \in Z$, there is a unique preference $R^{0} \in \mathcal{R}$ such that $p\left(R^{0}\right)=z$, and

(BS-iii) for each pair $x, y \in Z$ with $x \neq y$, there is a preference $R^{0} \in \mathcal{R}(y, x)$.

Conditions (i) and (ii) of our minimally rich domain are equivalent to conditions (BS-i) and (BS-ii), respectively. Condition (BS-iii) is weaker than condition (iii) of our minimally rich domain. Thus, any minimally rich domain is BS-minimally rich. ${ }^{20}$

The following are examples of minimally rich domains:

(1) Domain $\mathcal{R}^{\text {sym }}$ of symmetric single-peaked preferences,

(2) Domain $\mathcal{R}^{a} \subset \mathcal{R}^{\text {sin }}$ of preferences such that for each $z \in Z$, there is a unique preference $R^{0} \in \mathcal{R}^{a}$ with $p\left(R^{0}\right)=z$ such that for each pair $x, y \in Z$ with $x<z<y$, $x I^{0} y$ if and only if $(x-z)^{2}=2(y-z)^{2}$,

(3) Domain $\mathcal{R}^{b} \subset \mathcal{R}^{\text {sin }}$ of preferences such that for each $z \in Z$, there is a unique preference $R^{0} \in \mathcal{R}^{b}$ with $p\left(R^{0}\right)=z$ such that for each pair $x, y \in Z$ with $x<z<y$, $x I^{0} y$ if and only if $(x-z)^{2}=t(z)(y-z)^{2}$, where $t$ is an increasing function from $Z$ to $[1,2] .^{21}$

Let $\mathcal{R}^{M R}$ be the class of minimally rich domains.

\footnotetext{
${ }^{19} \mathbb{N}$ denotes the set of natural numbers.

${ }^{20} \mathrm{It}$ is an open question whether the converse implication holds or not. Thus, there could be a domain that is BS-minimally rich, but not minimally rich. However, note that our minimally rich domains still cover many important domains in their applications.

${ }^{21} \mathrm{~A}$ function $t$ is increasing if for each pair $z, z^{\prime} \in Z$ with $z \leq z^{\prime}, t(z) \leq t\left(z^{\prime}\right)$.
} 
A minimally rich domain is much smaller than the single-peaked domain. By the definition of sd-strategy-proofness, if a rule is sd-strategy-proof on a domain, then it is sd-strategy-proof on any subdomain. Thus, sd-strategy-proofness on a minimally rich domain is weaker than sd-strategy-proofness on the single-peaked domain. Hence, on a minimally rich domain, potentially, there exist more rules that are sd-strategy-proof and onto. However, our first result, presented below, says that the probabilistic generalized median rules are still the unique class of rules satisfying these two properties.

Theorem 1. A rule defined on a minimally rich domain is sd-strategy-proof and onto if and only if it is a probabilistic generalized median rule.

The proof of Theorem 1 is in the Appendix. For the deterministic model, Berga and Serizawa (2000) characterize the generalized median rules ${ }^{22}$ by strategyproofness and ontoness on a BS-minimally rich domain. Our result can be interpreted as a counterpart of theirs for the probabilistic model.

As we mentioned above, the domain of symmetric single-peaked preferences is minimally rich. Thus, we obtain the following corollary of Theorem 1.

Corollary 1. A rule defined on the domain of symmetric single-peaked preferences is sd-strategy-proof and onto if and only if it is a probabilistic generalized median rule.

For the deterministic model, Border and Jordan (1983) characterize the generalized median rules by strategy-proofness and ontoness on the symmetric singlepeaked domain. Corollary 1 is an extension of their result to the probabilistic model.

\subsection{Maximal domain}

Next, we ask how much we can enlarge a domain to allow for the existence of sd-strategy-proof rules that satisfy ontoness and the no-vetoer condition. The definition of a maximal domain is as follows.

Definition 3. A domain $\mathcal{R} \subset \mathcal{P}$ is maximal for a list of properties if

(i) there is a rule on $\mathcal{R}$ satisfying the properties, and

(ii) for each domain $\hat{\mathcal{R}}$ with $\mathcal{R} \subset \hat{\mathcal{R}} \subset \mathcal{P}$, no rule on $\hat{\mathcal{R}}$ satisfies the same properties.

Note that a maximal domain for a list of properties may not be unique. We consider domains that include a minimally rich domain.

\footnotetext{
${ }^{22}$ The generalized median rules are defined as follows. Let $a \equiv\left(a_{S}\right)_{S \in 2^{N}}$ be a collection of points in $Z$ such that (i) $a_{\emptyset}=\bar{z}$, (ii) $a_{N}=\underline{z}$, and (iii) for each $S, T \in 2^{N}, a_{S \cup T} \leq a_{S}$. This collection is the signature of the deterministic rule. Let $\mathcal{A}$ be the set of signatures.

Generalized median rule with signature $a \in \mathcal{A}, G \boldsymbol{M}^{a}$ : for each $R \in \mathcal{R}^{N}, G M^{a}(R)=$ $\min _{S \subseteq N}\left\{\max \left\{p\left(R_{i}\right)_{i \in S}, a_{S}\right\}\right\}$.

Let $D \equiv\left(D_{S}\right)_{S \in 2^{N}} \in \Delta$ be such that for each $S \in 2^{N}, D_{S}$ is a degenerate distribution. Let $a \equiv\left(a_{S}\right)_{S \in 2^{N}}$ be such that for each $S \in 2^{N}, a_{S}$ is the point at which $D_{S}$ places probability one. Then, the probabilistic generalized median rules $g^{D}$ with signature $D$ coincides with the generalized median rule $G M^{a}$ with signature $a \equiv\left(a_{S}\right)_{S \in 2^{N}}$.

In Berga and Serizawa (2000), this class is referred to as "generalized median voter schemes".
} 
Theorem 2. Assume that there are at least three agents, and let $\mathcal{R} \in \mathcal{R}^{M R}$. The domain of convex preferences is the unique maximal domain including $\mathcal{R}$ for sdstrategy-proofness, ontoness, and the no-vetoer condition.

The proof of Theorem 2 is in the Appendix.

Remark 1. Assume that there are only two agents. No rule defined on a minimally rich domain satisfies sd-strategy-proofness, ontoness, and the no-vetoer condition. ${ }^{23}$ Thus, in Theorem 2, we require that there be at least three agents.

Remark 2. Note that the random dictatorship rules ${ }^{24}$ satisfy sd-strategy-proofness and ontoness on the universal domain, but not the no-vetoer condition. Thus, in order to rule out such trivial rules, we impose the no-vetoer condition in Theorem 2. We remark that this condition also excludes some rules other than the random dictatorship rules. Furthermore, if we do not impose the no-vetoer condition, there are sd-strategy-proof and onto rules other than the random dictatorships on domains that include non-convex preferences. Thus, the no-vetoer condition is crucial to obtain convex preferences as a maximal domain. Example 1 below illustrates that, on a domain that include non-convex preferences, there is a non-random dictatorship rule that satisfies sd-strategy-proofness and ontoness, but not the no-vetoer condition. ${ }^{25}$

Example 1. A preference $R_{i}$ is outside convex if there are two intervals $\left[a^{-}\left(R_{i}\right), a^{+}\left(R_{i}\right)\right]$ and $\left[b^{-}\left(R_{i}\right), b^{+}\left(R_{i}\right)\right]$ of $Z$ such that $(1) a^{+}\left(R_{i}\right) \leq b^{-}\left(R_{i}\right),(2) p\left(R_{i}\right) \equiv\left[a^{-}\left(R_{i}\right), a^{+}\left(R_{i}\right)\right] \cup$ $\left[b^{-}\left(R_{i}\right), b^{+}\left(R_{i}\right)\right]$, and $(3)$ for each $X \in\left\{\left[\underline{z}, a^{+}\left(R_{i}\right)\right],\left[b^{-}\left(R_{i}\right), \bar{z}\right]\right\}$ and each $z \in X$, $U\left(R_{i}, z\right) \cap X$ is convex. Let $\mathcal{R}^{\text {ovex }}$ be the domain of outside convex preferences. Note that the domain $\mathcal{R}^{\text {ovex }}$ includes non-convex preferences and minimally rich domains. Let $\hat{f}:\left(\mathcal{R}^{\text {ovex }}\right)^{N} \rightarrow \Delta(Z)$ be the rule such that for each $R \in \hat{\mathcal{R}}^{N}$, and each $X \subset Z$,

$$
\hat{f}(R)(X)=\frac{1}{2} \cdot 1_{X}\left(\left\{\min _{i \in N} a^{+}\left(R_{i}\right)\right\}\right)+\frac{1}{2} \cdot 1_{X}\left(\left\{\max _{i \in N} b^{-}\left(R_{i}\right)\right\}\right) .
$$

Then, $\hat{f}$ is sd-strategy-proof and onto, but violates the no-vetoer condition. However, it is not the random dictatorship rules.

For the deterministic model, Berga and Serizawa (2000) prove that the convex domain is the unique maximal domain including a BS-minimally rich domain for

\footnotetext{
${ }^{23}$ To see this, suppose that there is a rule $f$ on $\mathcal{R} \in \mathcal{R}^{M R}$ satisfying these three properties. Let $N \equiv\{1,2\}$. Let $R_{1} \in \mathcal{R}$ be such that $p\left(R_{1}\right)<\bar{z}$, and for each $z \in Z, z R_{1} \bar{z}$. Let $R_{2} \in \mathcal{R}$ be such that $p\left(R_{2}\right) \equiv \bar{z}$. Then, by the no-vetoer condition, $f(R)(\{\bar{z}\})=1$. Let $\hat{R}_{1} \in \mathcal{R}$ be such that $p\left(\hat{R}_{1}\right) \equiv \underline{z}$. Then, by uncompromisingness (see Proposition 1 in Subsection 3.3), $f\left(\hat{R}_{1}, R_{2}\right)(\{\bar{z}\})=1$. Thus, $f\left(\hat{R}_{1}, R_{2}\right)(\{\underline{z}\}) \neq 1$. This contradicts the no-vetoer condition.

${ }^{24}$ Let $\mathcal{W}$ be the set of weights $w$ such that $w \equiv\left(w_{1}, \ldots, w_{n}\right) \in[0,1]^{N}$ and $\sum_{i \in N} w_{i}=1$.
} Random dictatorship rule with weight $\boldsymbol{w} \in \mathcal{W}, \boldsymbol{R} \boldsymbol{D}^{\boldsymbol{w}}$ : for each $R \in \mathcal{R}^{N}$ and each $X \subset Z$,

$$
R D^{w}(R)(X)=\sum_{i \in N} w_{i} \cdot 1_{X}\left(p\left(R_{i}\right)\right)
$$

\footnotetext{
${ }^{25}$ For the deterministic model, similar arguments are found in Berga and Serizawa (2000).
} 
strategy-proofness, ontoness, and the BS-no-vetoer condition. For the probabilistic model, it is easy to check if there is a rule that satisfy these three properties on the convex domain. Furthermore, the BS-no-vetoer condition together with sd-strategyproofness implies the no-vetoer condition. Thus, as a corollary of Theorem 2, we obtain a counterpart of their result for the probabilistic case.

Corollary 2. Assume that there are at least three agents. The domain of convex preferences is the unique maximal domain including a minimally rich domain for sd-strategy-proofness, ontoness, and the BS-no-vetoer condition.

Since each minimally rich domain is a subclass of the single-peaked domain, each domain that includes the single-peaked domain also includes a minimally rich domain. Thus, the class of domains that include the single-peaked domain is a subclass of the class of domains that include a minimally rich domain. We obtain the following as a corollary of Theorem 2 .

Corollary 3. Assume that there are at least three agents. The domain of convex preferences is the unique maximal domain including the single-peaked domain for sd-strategy-proofness, ontoness, and the no-vetoer condition.

Since the symmetric single-peaked domain is a minimally rich domain, we also obtain the following corollary.

Corollary 4. Assume that there are at least three agents. The domain of convex preferences is the unique maximal domain including the symmetric single-peaked domain for sd-strategy-proofness, ontoness, and the no-vetoer condition.

Remark 3. A deterministic alternative can be regarded as a degenerate probability distribution. Also, each of the properties introduced in our paper, when imposed on deterministic rules, reduces to the property of the same name for the deterministic model. Thus, as a byproduct of our results, for the deterministic model, we also obtain results that are close to the results of Berga and Serizawa (2000).

\subsection{Technical discussions: uncompromisingness and richness of domains}

We now discuss some key points of our results and proofs. First, we introduce a property that plays an important role in the proof of the uniqueness part of Theorem 1. This notion was first introduced by Ehlers et al. (2002): when an agent's preference changes, the chosen distribution does not change outside the interval whose endpoints are his initial peak and new peak. ${ }^{26}$

Given $R_{i}, \hat{R}_{i} \in \mathcal{R}$, let $E\left(R_{i}, \hat{R}_{i}\right) \equiv\left[\min \left\{p\left(R_{i}\right), p\left(\hat{R}_{i}\right)\right\}, \max \left\{p\left(R_{i}\right), p\left(\hat{R}_{i}\right)\right\}\right]$.

Uncompromisingness: For each $R \in \mathcal{R}^{N}$, each $i \in N$, each $\hat{R}_{i} \in \mathcal{R}$, and each $X \subset Z$ such that $X \cap E\left(R_{i}, \hat{R}_{i}\right)=\emptyset, f(R)(X)=f\left(\hat{R}_{i}, R_{-i}\right)(X)$.

The next proposition is key to Theorem 1.

Proposition 1. On a minimally rich domain, sd-strategy-proofness and ontoness imply uncompromisingness.

\footnotetext{
${ }^{26}$ This property is an extension to the probabilistic model of a property introduced for the deterministic model by Border and Jordan (1983).
} 
The proof is in the Appendix. Ehlers et al. (2002) establish that on the single-peaked domain, sd-strategy-proofness and peak-onlyness imply uncompromisingness. ${ }^{27}$ Note that, by the definition of a minimally rich domain, any rule defined on a minimally rich domain is peak-only. Thus, the assumption of our Proposition 1 is stronger than theirs. However, we cannot directly apply their result and proof techniques to prove Proposition 1. As we explain below, there is a crucial difference between the single-peaked domain and a minimally rich domain.

We now introduce a new property of rules. This property is implied by uncompromisingness. Hereafter, we only focus on this property to simplify our subsequent argument.

Invariance property: For each $R \in \mathcal{R}^{N}$, each $i \in N$, each $\hat{R}_{i} \in \mathcal{R}$, and each pair $a, b \in Z$ such that $a<p\left(R_{i}\right)<p\left(\hat{R}_{i}\right)<b, f(R)([a, b])=f\left(\hat{R}_{i}, R_{-i}\right)([a, b])$.

Next, we introduce another richness condition on a domain. A domain $\mathcal{R} \subset \mathcal{P}$ is rich* $^{*}$ if for each $R_{i} \in \mathcal{R}$, and each pair $a, b \in Z$ with $a<p\left(R_{i}\right)<b$, there is $R_{i}^{\prime} \in \mathcal{R}$ such that (i) $p\left(R_{i}\right)=p\left(R_{i}^{\prime}\right)$, and (ii) $U\left(R_{i}^{\prime}, b\right)=[a, b]$. Note that the single-peaked domain is rich* ${ }^{*}$. Under this richness condition, we can directly apply the proof techniques developed by Ehlers et al. (2002) to obtain the invariance property, as shown in the following fact.

Fact 1. Let $\mathcal{R} \subseteq \mathcal{R}^{\text {sin }}$ be a rich* domain. If a rule $f$ on $\mathcal{R}^{N}$ is sd-strategy-proof and peak-only, then it satisfies the invariance property.

Proof. ${ }^{28}$ Let $R \in \mathcal{R}^{N}, i \in N, \hat{R}_{i} \in \mathcal{R}$, and $a, b \in Z$ be such that $a<p\left(R_{i}\right)<$ $p\left(\hat{R}_{i}\right)<b$. Since $\mathcal{R}$ is rich $^{*}$, there are $R_{i}^{\prime}, \bar{R}_{i} \in \mathcal{R}$ such that (i) $p\left(R_{i}\right)=p\left(R_{i}^{\prime}\right)$, $p\left(\hat{R}_{i}\right)=p\left(\bar{R}_{i}\right)$, and (ii) $U\left(R_{i}^{\prime}, b\right)=[a, b]=U\left(\bar{R}_{i}, b\right)$. Then, by sd-strategy-proofness, $f\left(R_{i}^{\prime}, R_{-i}\right)\left(U\left(R_{i}^{\prime}, b\right)\right) \geq f\left(\bar{R}_{i}, R_{-i}\right)\left(U\left(R_{i}^{\prime}, b\right)\right)$. Similarly, by sd-strategy-proofness, $f\left(R_{i}^{\prime}, R_{-i}\right)\left(U\left(\bar{R}_{i}, b\right)\right) \leq f\left(\bar{R}_{i}, R_{-i}\right)\left(U\left(\bar{R}_{i}, b\right)\right)$. Since $U\left(R_{i}^{\prime}, b\right)=[a, b]=U\left(\bar{R}_{i}, b\right)$, we have $f\left(R_{i}^{\prime}, R_{-i}\right)([a, b])=f\left(\bar{R}_{i}, R_{-i}\right)([a, b])$. Finally, by peak-onlyness, $f(R)([a, b])=$ $f\left(R_{i}^{\prime}, R_{-i}\right)([a, b])$ and $f\left(\hat{R}_{i}, R_{-i}\right)([a, b])=f\left(\bar{R}_{i}, R_{-i}\right)([a, b])$. Thus, $f(R)([a, b])=$ $f\left(\hat{R}_{i}, R_{-i}\right)([a, b])$.

On the other hand, since no two distinct preferences have the same peak on any minimally rich domain, no minimally rich domain is rich* ${ }^{*}$ Thus, we cannot apply the argument used in the proof of Fact 1 to obtain the invariance property on a minimally rich domain. This is the main difference between the single-peaked domain and a minimally rich domain, and the reason the proof techniques of Ehlers et al. (2002) cannot be applied to prove Proposition 1.

We develop two lemmas to overcome this difficulty. These lemmas give us variants of the invariance property. Lemma 1 says that under sd-strategy-proofness and ontoness, if a group of agents with the lowest peak, say $p^{1}$, changes their peaks, and their new peak point, say $\bar{p}$, is still less than the second lowest peak, then the outcome distribution does not change outside the interval with endpoints $p^{1}$ and $\bar{p}$. A similar statement applies to a group of agents with the highest peaks.

\footnotetext{
${ }^{27}$ See Ehlers et al. (2002, Lemma 3.1). They also establish that peak-onlyness is implied by sd-strategy-proof and unanimity (see Ehlers et al., 2002, Proposition 5.2).

${ }^{28}$ Indeed, in this proof, we use the proof techniques developed by Ehlers et al. (2002).
} 
Lemma 2 says that under sd-strategy-proofness and ontoness, for each point $\bar{p} \in Z$, if a group of agents whose peaks are less than $\bar{p}$ changes their peaks to $\bar{p}$, then the outcome distribution does not change outside the interval with endpoints $p^{1}$ and $\bar{p}$. Also, a similar statement applies to a group of agents whose peaks are greater than $\bar{p}$.

Furthermore, on a minimally rich domain, ontoness is indispensable to obtain uncompromisingness ${ }^{29}$ while it is not required on the single-peaked domain. This is also one of the differences between the single-peaked domain and minimally rich domains.

Since the characterization result of the class of sd-strategy-proof and onto rules on a minimally rich domain is utilized in the proof of our maximal domain theorem, proving Proposition 1 and Theorem 1 is also key to Theorem 2.

\section{Concluding remarks}

We established that, on a minimally rich domain, the probabilistic generalized median rules are the unique class of sd-strategy-proof and onto rules. Recently, by using the characterization result of Berga and Serizawa (2000), Massó and Moreno de Barreda (2011) characterize the class of strategy-proof deterministic rules on the symmetric single-peaked domain. ${ }^{30}$ It is an interesting question whether a result parallel to theirs can be obtained for the probabilistic model.

Next, we examined how much we can enlarge a domain to allow for the existence of rules that satisfy sd-strategy-proofness, ontoness, and the no-vetoer condition. We established that the domain of convex preferences is the unique maximal domain including a minimally rich domain for these properties. For the deterministic model, Berga and Serizawa (2000) establish that the unique maximal domain including a BS-minimally rich domain for strategy-proofness, ontoness, and the BS-no-vetoer condition is the convex domain. Our result can be interpreted as an extension of theirs to the probabilistic model.

In this paper, we focused on the analysis of the probabilistic rules defined on the set of ordinal preferences of agents. Another possible formulation of probabilistic rules is to define a rule on the set of von Neumann-Morgenstern utility functions, and assume that agents compare probability distributions based on their expected utilities. Note that the notion of sd-strategy-proofness is different from that of strategy-proofness based on the expected utility. To see the difference between the two notions of strategy-proofness for probabilistic rules, it is also interesting to examine the conditions on the domains of rules that satisfy strategy-proofness based on the expected utility.

\footnotetext{
${ }^{29}$ For example, for the deterministic model, strategy-proofness alone does not imply uncompromisingness on the symmetric single-peaked domain (Border and Jordan, 1983). Since the class of probabilistic rules includes deterministic rules as a special case, their example also holds for the probabilistic model.

${ }^{30}$ This class of rules is much larger than the class of generalized median rules.
} 


\section{Appendix: Proofs}

\subsection{Preliminary results}

Let $\underline{\mathcal{R}} \in \mathcal{R}^{M R}$, and let $\mathcal{R}$ be a domain such that $\underline{\mathcal{R}} \subseteq \mathcal{R} \subseteq \mathcal{R}^{\text {sin }}$. Given $R_{i} \in \mathcal{R}$ and $z \in Z$, the strict upper contour set of $\boldsymbol{R}_{\boldsymbol{i}}$ at $\boldsymbol{z}$ is the set $\boldsymbol{S U}\left(\boldsymbol{R}_{\boldsymbol{i}}, \boldsymbol{z}\right)$ $\equiv\left\{y \in Z: y P_{i} z\right\}$. Fact 2 says that the stochastic dominance relation $R_{i}^{s d}$ is equivalent to that defined in terms of strict upper contour sets.

Fact 2 (Ehlers et al., 2002, Lemma 2.1). For each $R_{i} \in \mathcal{R}$, and each pair $Q$, $Q^{\prime} \in \Delta(Z), Q R_{i}^{s d} Q^{\prime}$ if and only if for each $z \in Z, Q\left(S U\left(R_{i}, z\right)\right) \geq Q^{\prime}\left(S U\left(R_{i}, z\right)\right)$, and $Q P_{i}^{s d} Q^{\prime}$ if and only if $Q R_{i}^{s d} Q^{\prime}$ and for some $z \in Z, Q\left(S U\left(R_{i}, z\right)\right)>$ $Q^{\prime}\left(S U\left(R_{i}, z\right)\right)$.

Given $R \in \mathcal{R}^{N}$, let $p(R) \equiv \min _{i \in N}\left\{p\left(R_{i}\right)\right\}$, and $\bar{p}(R) \equiv \max _{i \in N}\left\{p\left(R_{i}\right)\right\}$, and let $E(R) \equiv[p(R), \bar{p}(R)]$, that is, $E(R)$ is the set of ex-post efficient outcomes for $R$. Fact 3 says that sd-efficiency is equivalent to ex-post efficiency.

Fact 3 (Ehlers et al., 2002, Lemma 2.2). Let $f$ be a rule on $\mathcal{R}^{N}$. Then, $f$ is sd-efficient if and only if for each $R \in \mathcal{R}^{N}, f(R)(E(R))=1$.

Fact 4 says that sd-strategy-proofness and unanimity imply ex-post efficiency.

Fact 4 (Ehlers et al., 2002, Proposition 5.1). Let $f$ be an sd-strategy-proof and unanimous rule on $\mathcal{R}^{N}$. Then, for each $R \in \mathcal{R}^{N}, f(R)(E(R))=1$.

Fact 5 says that sd-strategy-proofness and ontoness imply unanimity.

Fact 5. Let $f$ be an sd-strategy-proof and onto rule on $\mathcal{R}^{N}$. Then, it is unanimous.

Proof of Fact 5. Let $y \in Z$. Let $\bar{R} \in \mathcal{R}^{N}$ be such that for each $i \in N$, $p\left(\bar{R}_{i}\right) \equiv y$. We show that $f(\bar{R})(\{y\})=1$. By ontoness, there is $R \in \mathcal{R}^{N}$ such that $f(R)(\{y\})=1$. By sd-strategy-proofness, $f\left(\bar{R}_{1}, R_{-1}\right)\left(U\left(\bar{R}_{1}, y\right)\right) \geq f(R)\left(U\left(\bar{R}_{1}, y\right)\right)$. Since $U\left(\bar{R}_{1}, y\right)=\{y\}$ and $f(R)(\{y\})=1$, then $f\left(\bar{R}_{1}, R_{-1}\right)(\{y\})=1$. Similarly, by sd-strategy-proofness, $f\left(\bar{R}_{1,2}, R_{-1,2}\right)\left(U\left(\bar{R}_{2}, y\right)\right) \geq f\left(\bar{R}_{1}, R_{-1}\right)\left(U\left(\bar{R}_{2}, y\right)\right)$. Since $U\left(\bar{R}_{2}, y\right)=\{y\}$ and $f\left(\bar{R}_{1}, R_{-1}\right)(\{y\})=1, f\left(\bar{R}_{1,2}, R_{-1,2}\right)(\{y\})=1$. Repeating this argument for agents $j=3, \ldots n$, we have $f(\bar{R})(\{y\})=1$.

Remark 4. From facts 3,4 , and 5 , if a rule $f$ on $\mathcal{R}^{N}$ is sd-strategy-proof and onto, then it is sd-efficient.

\subsection{Proof of Theorem 1.}

First, we prove two lemmas. Next, we prove Proposition 1 . Then, we prove Theorem 1. Let $\mathcal{R} \in \mathcal{R}^{M R}$. Given $R \in \mathcal{R}^{N}$ and $\ell \in\{1, \ldots, \bar{n}(R)\}$, let $N^{\ell}(R)$ be the set of agents whose peak is equal to the $\ell$-th smallest peak amount at $R$, that is $N^{\ell}(R) \equiv\left\{i \in N: p\left(R_{i}\right)=p^{\ell}(R)\right\}$.

Lemma 1. Let $f$ be an sd-strategy-proof and onto rule on $\mathcal{R}^{N}$. Let $R \in \mathcal{R}^{N}, \bar{N} \subseteq$ $N^{1}(R), \bar{R}_{\bar{N}} \in \mathcal{R}^{N}$, and $\bar{p}, x \in Z$ be such that (1-1) for each $i \in \bar{N}, p\left(\bar{R}_{i}\right)=\bar{p}$, (1-2) $\bar{p}<x$, and (1-3) $p^{1}(R)<\bar{p} \leq p^{2}(R)$. Then, $f(R)\left(\left[p^{1}(R), x[)=f\left(\bar{R}_{\bar{N}}, R_{-\bar{N}}\right)\left(\left[p^{1}(R), x[)\right.\right.\right.\right.$.

Proof of Lemma 1. Let $x^{0} \equiv p^{1}(R)$. By condition (iii) of the minimally rich domain in Definition 1 , there exist a finite $K \in \mathbb{N}$ and a sequence $\left\{R_{0}^{(k)}\right\}_{k=1}^{K}$ of 
preferences in $\mathcal{R}$ such that (i) $x^{0} P_{0}^{(1)} x$ and $x^{0}<p\left(R_{0}^{(1)}\right)<x$, (ii) for each $k \in$ $\{2, \ldots, K\}, p\left(R_{0}^{(k-1)}\right) P_{0}^{(k)} x$, and $p\left(R_{0}^{(k-1)}\right)<p\left(R_{0}^{(k)}\right)<x$, and (iii) $p\left(R_{0}^{(K-1)}\right)<$ $\bar{p} \leq p\left(R_{0}^{(K)}\right)$. For each $k \in\{2, \ldots, K\}$, let $x^{k} \equiv p\left(R_{0}^{(k)}\right)$.

Step 1. For each $k \in\{1, \ldots, K\}$, each $\bar{R} \in \mathcal{R}^{N}$, each $\bar{N} \subseteq N^{1}(\bar{R})$, and each $\tilde{R}_{\bar{N}} \in \mathcal{R}^{\bar{N}}$, if (a) $p^{1}(\bar{R})=x^{0}$, (b) for each $i \in \bar{N}, \tilde{R}_{i} \equiv R_{0}^{(k)}$, and (c) $x^{k-1} \leq p^{2}(\bar{R})$, then, $f(\bar{R})\left(\left[x^{0}, x[)=f\left(\tilde{R}_{\bar{N}}, \bar{R}_{-\bar{N}}\right)\left(\left[x^{0}, x[)\right.\right.\right.\right.$.

Proof of Step 1. We prove Step 1 by induction on $k$. Assume that $k=1$. Let $\bar{R} \in \mathcal{R}^{N}, \bar{N} \subseteq N^{1}(\bar{R})$, and $\tilde{R}_{\bar{N}} \in \mathcal{R}^{\bar{N}}$ be such that (a) $p^{1}(\bar{R})=x^{0}$, (b) for each $i \in$ $\bar{N}, \tilde{R}_{i} \equiv R_{0}^{(1)}$, and (c) $x^{0} \leq p^{2}(\bar{R})$. Without loss of generality, let $\bar{N} \equiv\left\{1, \ldots, \bar{n}^{1}\right\}$. First, we replace the preference $\bar{R}_{i}$ of agent $i \in \bar{N}$ with the preference $\tilde{R}_{i}$, inductively. Note that, by sd-efficiency, $f(\bar{R})\left(\left[\underline{z}, x^{0}[)=f\left(\tilde{R}_{1}, \bar{R}_{-1}\right)\left(\left[\underline{z}, x^{0}[)=0\right.\right.\right.\right.$. Then, ${ }^{31}$

$$
\begin{aligned}
f(\bar{R})\left(\left[x^{0}, x[)\right.\right. & =f(\bar{R})\left(S U\left(\bar{R}_{1}, x\right)\right) \text { by } s d-E \\
& \geq f\left(\tilde{R}_{1}, \bar{R}_{-1}\right)\left(S U\left(\bar{R}_{1}, x\right)\right) \text { by } s d-S P \text { and Fact } 2 \\
& =f\left(\tilde{R}_{1}, \bar{R}_{-1}\right)\left(\left[x^{0}, x[) \text { by } s d-E .\right.\right.
\end{aligned}
$$

Conversely,

$$
\begin{aligned}
f\left(\tilde{R}_{1}, \bar{R}_{-1}\right)\left(\left[x^{0}, x[)\right.\right. & =f\left(\tilde{R}_{1}, \bar{R}_{-1}\right)\left(S U\left(\tilde{R}_{1}, x\right)\right) \text { by } s d-E \text { and } x^{0} \tilde{P}_{1} x \\
& \geq f(\bar{R})\left(S U\left(\tilde{R}_{1}, x\right)\right) \text { by } s d-S P \text { and Fact } 2 \\
& =f(\bar{R})\left(\left[x^{0}, x[) \text { by } s d-E \text { and } x^{0} \tilde{P}_{1} x .\right.\right.
\end{aligned}
$$

Thus, $f(\bar{R})\left(\left[x^{0}, x[)=f\left(\tilde{R}_{1}, \bar{R}_{-1}\right)\left(\left[x^{0}, x[)\right.\right.\right.\right.$. Since $\tilde{R}_{1}=\tilde{R}_{2}$, by applying the same argument to the profile $\left(\tilde{R}_{1}, \bar{R}_{-1}\right)$, we obtain $f\left(\tilde{R}_{1}, \bar{R}_{-1}\right)\left(\left[x^{0}, x[)=f\left(\tilde{R}_{1,2}, \bar{R}_{-1,2}\right)\left(\left[x^{0}, x[)\right.\right.\right.\right.$. Repeating this argument for agents $j=3, \ldots, \bar{n}^{1}, f(\bar{R})\left(\left[x^{0}, x[)=f\left(\widetilde{R}_{\bar{N}}, \bar{R}_{-\bar{N}}\right)\left(\left[x^{0}, x[)\right.\right.\right.\right.$. Thus, Step 1 holds if $k=1$.

Next, we assume that $k \geq 2$. Let $\bar{R} \in \mathcal{R}^{N}, \bar{N} \subseteq N^{1}(\bar{R})$, and $\tilde{R}_{\bar{N}} \in \mathcal{R}^{\bar{N}}$ be such that (a) $p^{1}(\bar{R})=x^{0}$, (b) for each $i \in \bar{N}, \tilde{R}_{i} \equiv R_{0}^{(k)}$, and (c) $x^{k-1} \leq p^{2}(\bar{R})$. Without loss of generality, let $\bar{N} \equiv\left\{1, \ldots, \bar{n}^{k}\right\}$.

As the induction hypothesis, we assume that

A: For each $\hat{R} \in \mathcal{R}^{N}$, each $\hat{N} \subseteq N^{1}(\hat{R})$, and each $R_{\hat{N}}^{\prime} \in \mathcal{R}^{\hat{N}}$, if $(A-a) p^{1}(\hat{R})=x^{0}$, (A-b) for each $i \in \hat{N}, R_{i}^{\prime}=R_{0}^{(k-1)}$, and $(A-c) x^{k-2} \leq p^{2}(\hat{R})$, then, $f(\hat{R})\left(\left[x^{0}, x[)=\right.\right.$ $f\left(R_{\hat{N}}^{\prime}, \hat{R}_{-\hat{N}}\right)\left(\left[x^{0}, x[)\right.\right.$.

We show that $f(\bar{R})\left(\left[x^{0}, x[)=f\left(\tilde{R}_{\bar{N}}, \bar{R}_{-\bar{N}}\right)\left(\left[x^{0}, x[)\right.\right.\right.\right.$. For each $i \in N^{1}(\bar{R})$, let $R_{i}^{\prime} \equiv R_{0}^{(k-1)}$. Then, by induction hypothesis (A), ${ }^{32}$

$$
f(\bar{R})\left(\left[x^{0}, x[)=f\left(R_{N^{1}(\bar{R})}^{\prime}, \bar{R}_{-N^{1}(\bar{R})}\right)\left(\left[x^{0}, x[) .\right.\right.\right.\right.
$$

\footnotetext{
${ }^{31}$ Hereafter, we occasionally abbreviate $s d$-efficiency and $s d$-strategy-proofness as $s d$-E and $s d$ $S P$, respectively.

${ }^{32}$ Since (a) $p^{1}(\bar{R})=x^{0}$ and (c) $x^{k-1} \leq p^{2}(\bar{R})$, all the assumptions (A-a), (A-b), and (A-c) of induction hypothesis (A) are satisfied.
} 
Next, we replace the preference $R_{i}^{\prime}$ of agent $i \in \bar{N}$ with the preference $\tilde{R}_{i}$, inductively. Note that, by sd-efficiency and $x^{k-1} \leq p^{2}(\bar{R}), f\left(R_{N^{1}(\bar{R})}^{\prime}, \bar{R}_{-N^{1}(\bar{R})}\right)\left(\left[\underline{z}, x^{k-1}[)=\right.\right.$ $f\left(\tilde{R}_{1}, R_{N^{1}(\bar{R}) \backslash\{1\}}^{\prime}, \bar{R}_{-N^{1}(\bar{R})}\right)\left(\left[\underline{z}, x^{k-1}[)=0\right.\right.$. Then,

$$
\begin{aligned}
f\left(R_{N^{1}(\bar{R})}^{\prime}, \bar{R}_{-N^{1}(\bar{R})}\right) & \left(\left[x^{0}, x[)\right.\right. \\
& =f\left(R_{N^{1}(\bar{R})}^{\prime}, \bar{R}_{-N^{1}(\bar{R})}\right)\left(\left[x^{k-1}, x[) \text { by } s d-E\right.\right. \\
& =f\left(R_{N^{1}(\bar{R})}^{\prime}, \bar{R}_{-N^{1}(\bar{R})}\right)\left(S U\left(R_{1}^{\prime}, x\right)\right) \text { by } s d-E \\
& \geq f\left(\tilde{R}_{1}, R_{N^{1}(\bar{R}) \backslash\{1\}}^{\prime}, \bar{R}_{-N^{1}(\bar{R})}\right)\left(S U\left(R_{1}^{\prime}, x\right)\right) \text { by } s d-S P \text { and Fact } 2 \\
& =f\left(\tilde{R}_{1}, R_{N^{1}(\bar{R}) \backslash\{1\}}^{\prime}, \bar{R}_{-N^{1}(\bar{R})}\right)\left(\left[x^{k-1}, x[) \text { by } s d-E\right.\right. \\
& =f\left(\tilde{R}_{1}, R_{N^{1}(\bar{R}) \backslash\{1\}}^{\prime}, \bar{R}_{-N^{1}(\bar{R})}\right)\left(\left[x^{0}, x[)\right.\right. \text { by sd-E. }
\end{aligned}
$$

Conversely,

$$
\begin{aligned}
f\left(\tilde{R}_{1}, R_{N^{1}(\bar{R}) \backslash\{1\}}^{\prime}\right. & \left.\bar{R}_{-N^{1}(\bar{R})}\right)\left(\left[x^{0}, x[)\right.\right. \\
& =f\left(\tilde{R}_{1}, R_{N^{1}(\bar{R}) \backslash\{1\}}^{\prime}, \bar{R}_{-N^{1}(\bar{R})}\right)\left(\left[x^{k-1}, x[) \text { by } s d-E\right.\right. \\
& =f\left(\tilde{R}_{1}, R_{N^{1}(\bar{R}) \backslash\{1\}}^{\prime}, \bar{R}_{-N^{1}(\bar{R})}\right)\left(S U\left(\tilde{R}_{1}, x\right)\right) \text { by } s d-E \text { and } x^{k-1} \tilde{P}_{1} x \\
& \geq f\left(R_{N^{1}(\bar{R})}^{\prime}, \bar{R}_{-N^{1}(\bar{R})}\right)\left(S U\left(\tilde{R}_{1}, x\right)\right) \text { by } s d-S P \text { and Fact } 2 \\
& =f\left(R_{N^{1}(\bar{R})}^{\prime}, \bar{R}_{-N^{1}(\bar{R})}\right)\left(\left[x^{k-1}, x[) \text { by } s d-E \text { and } x^{k-1} \tilde{P}_{1} x\right.\right. \\
& =f\left(R_{N^{1}(\bar{R})}^{\prime}, \bar{R}_{-N^{1}(\bar{R})}\right)\left(\left[x^{0}, x[) \text { by } s d-E .\right.\right.
\end{aligned}
$$

Thus, $f\left(R_{N^{1}(\bar{R})}^{\prime}, \bar{R}_{-N^{1}(\bar{R})}\right)\left(\left[x^{0}, x[)=f\left(\tilde{R}_{1}, R_{N^{1}(\bar{R}) \backslash\{1\}}^{\prime}, \bar{R}_{-N^{1}(\bar{R})}\right)\left(\left[x^{0}, x[)\right.\right.\right.\right.$. Since $\tilde{R}_{1}=$ $\tilde{R}_{2}$, by applying the same argument to the profile $\left(\tilde{R}_{1}, R_{N^{1}(\bar{R}) \backslash\{1\}}^{\prime}, \bar{R}_{-N^{1}(\bar{R})}\right)$, we have $f\left(\tilde{R}_{1}, R_{N^{1}(\bar{R}) \backslash\{1\}}^{\prime}, \bar{R}_{-N^{1}(\bar{R})}\right)\left(\left[x^{0}, x[)=f\left(\tilde{R}_{1,2}, R_{N^{1}(\bar{R}) \backslash\{1,2\}}^{\prime}, \bar{R}_{-N^{1}(\bar{R})}\right)\left(\left[x^{0}, x[)\right.\right.\right.\right.$. Repeating this argument for agents $j=3, \ldots, \bar{n}^{k}$,

$$
f\left(R_{N^{1}(\bar{R})}^{\prime}, \bar{R}_{-N^{1}(\bar{R})}\right)\left(\left[x^{0}, x[)=f\left(\tilde{R}_{\bar{N}}, R_{N^{1}(\bar{R}) \backslash \bar{N}}^{\prime}, \bar{R}_{-N^{1}(\bar{R})}\right)\left(\left[x^{0}, x[) .\right.\right.\right.\right.
$$

Therefore, if $\bar{N}=N^{1}(\bar{R})$, then Step 1 follows from (1) and (2). Thus, we assume that $\bar{N} \neq N^{1}(\bar{R})$, that is, $N^{1}(\bar{R}) \backslash \bar{N} \neq \emptyset$. Next, we consider the profile $\left(\tilde{R}_{\bar{N}}, \bar{R}_{-\bar{N}}\right)$. Then, $p^{2}\left(\tilde{R}_{\bar{N}}, \bar{R}_{-\bar{N}}\right)=\min \left\{x^{k}, p^{2}(\bar{R})\right\}$. Thus, $x^{k-2}<x^{k-1} \leq p^{2}\left(\tilde{R}_{\bar{N}}, \bar{R}_{-\bar{N}}\right)$. Note that $N^{1}\left(\tilde{R}_{\bar{N}}, \bar{R}_{-\bar{N}}\right)=N^{1}(\bar{R}) \backslash \bar{N} \neq \emptyset$, and $p^{1}\left(\tilde{R}_{\bar{N}}, \bar{R}_{-\bar{N}}\right)=p^{1}(\bar{R})=x^{0}$. Thus, the assumptions of induction hypothesis (A) also hold for the profile $\left(\tilde{R}_{\bar{N}}, \bar{R}_{-\bar{N}}\right)$. Then, by induction hypothesis (A),

$$
f\left(\tilde{R}_{\bar{N}}, \bar{R}_{-\bar{N}}\right)\left(\left[x^{0}, x[)=f\left(\tilde{R}_{\bar{N}}, R_{N^{1}(\bar{R}) \backslash \bar{N}}^{\prime}, \bar{R}_{-N^{1}(\bar{R})}\right)\left(\left[x^{0}, x[) .\right.\right.\right.\right.
$$

Thus, from (1), (2), and (3),

$$
f(\bar{R})\left(\left[x^{0}, x[)=f\left(\tilde{R}_{\bar{N}}, \bar{R}_{-\bar{N}}\right)\left(\left[x^{0}, x[) .\right.\right.\right.\right.
$$


Step 2. For each $k \in\{1, \ldots, K\}$, each $\bar{R} \in \mathcal{R}^{N}$, each $\bar{N} \subseteq N^{1}(\bar{R})$, each $\hat{p} \in$ ]$\left.x^{k-1}, x^{k}\right]$, and each $\hat{R}_{\bar{N}} \in \mathcal{R}^{\bar{N}}$, if (a) $p^{1}(\bar{R})=x^{0}$, (b) for each $i \in \bar{N}, p\left(\hat{R}_{i}\right)=\hat{p}$, and $(c) \hat{p} \leq p^{2}(\bar{R})$, then, $f(\bar{R})\left(\left[x^{0}, x[)=f\left(\hat{R}_{\bar{N}}, \bar{R}_{-\bar{N}}\right)\left(\left[x^{0}, x[)\right.\right.\right.\right.$.

Proof of Step 2. Let $\left.\left.k \in\{1, \ldots, K\}, \bar{R} \in \mathcal{R}^{N}, \bar{N} \subseteq N^{1}(\bar{R}), \hat{p} \in\right] x^{k-1}, x^{k}\right]$, and $\hat{R}_{\bar{N}} \in \mathcal{R}^{\bar{N}}$ be such that (a) $p^{1}(\bar{R})=x^{0}$, (b) for each $i \in \bar{N}, p\left(\hat{R}_{i}\right)=\hat{p}$, and (c) $\hat{p} \leq p^{2}(\bar{R})$. Without loss of generality, let $\bar{N} \equiv\left\{1, \ldots, \bar{n}^{k}\right\}$. If $\hat{p}=x^{k}$, then the desired conclusion follows from Step 1. Thus, we assume that $x^{k-1}<\hat{p}<x^{k}$.

For each $i \in N^{1}(\bar{R})$, let $\tilde{R}_{i} \equiv R_{0}^{(k)}$. Then, by Step 1 ,

$$
f(\bar{R})\left(\left[x^{0}, x[)=f\left(\tilde{R}_{N^{1}(\bar{R})}, \bar{R}_{-N^{1}(\bar{R})}\right)\left(\left[x^{0}, x[) .\right.\right.\right.\right.
$$

Next, we replace the preference $\tilde{R}_{i}$ of agent $i \in \bar{N}$ with the preference $\hat{R}_{i}$, inductively. Note that, by sd-efficiency and $\hat{p} \leq p^{1}\left(\tilde{R}_{N^{1}(\bar{R})}, \bar{R}_{-N^{1}(\bar{R})}\right), f\left(\tilde{R}_{N^{1}(\bar{R})}, \bar{R}_{-N^{1}(\bar{R})}\right)([\underline{z}, \hat{p}[)=$ $f\left(\hat{R}_{1}, \tilde{R}_{N^{1}(\bar{R}) \backslash\{1\}}, \bar{R}_{-N^{1}(\bar{R})}\right)([\underline{z}, \hat{p}[)=0$. Then,

$$
\begin{aligned}
f\left(\tilde{R}_{N^{1}(\bar{R})}, \bar{R}_{-N^{1}(\bar{R})}\right) & \left(\left[x^{0}, x[)\right.\right. \\
& =f\left(\tilde{R}_{N^{1}(\bar{R})}, \bar{R}_{-N^{1}(\bar{R})}\right)([\hat{p}, x[) \text { by } s d-E \\
& =f\left(\tilde{R}_{N^{1}(\bar{R})}, \bar{R}_{-N^{1}(\bar{R})}\right)\left(S U\left(\tilde{R}_{1}, x\right)\right) \text { by } s d-E \text { and } x^{k-1} \tilde{P}_{1} x \\
& \geq f\left(\hat{R}_{1}, \tilde{R}_{N^{1}(\bar{R}) \backslash\{1\}}, \bar{R}_{-N^{1}(\bar{R})}\right)\left(S U\left(\tilde{R}_{1}, x\right)\right) \text { by } s d-S P \text { and Fact } 2 \\
& =f\left(\hat{R}_{1}, \tilde{R}_{N^{1}(\bar{R}) \backslash\{1\}}, \bar{R}_{-N^{1}(\bar{R})}\right)\left(\left[\hat{p}, x[) \text { by } s d-E \text { and } x^{k-1} \tilde{P}_{1} x\right.\right. \\
& =f\left(\hat{R}_{1}, \tilde{R}_{N^{1}(\bar{R}) \backslash\{1\}}, \bar{R}_{-N^{1}(\bar{R})}\right)\left(\left[x^{0}, x[) \text { by } s d-E .\right.\right.
\end{aligned}
$$

Conversely,

$$
\begin{aligned}
f\left(\hat{R}_{1}, \tilde{R}_{N^{1}(\bar{R}) \backslash\{1\}}, \bar{R}_{-N^{1}(\bar{R})}\right) & \left(\left[x^{0}, x[)\right.\right. \\
& =f\left(\hat{R}_{1}, \tilde{R}_{N^{1}(\bar{R}) \backslash\{1\}}, \bar{R}_{-N^{1}(\bar{R})}\right)([\hat{p}, x[) \text { by } s d-E \\
& =f\left(\hat{R}_{1}, \tilde{R}_{N^{1}(\bar{R}) \backslash\{1\}}, \bar{R}_{-N^{1}(\bar{R})}\right)\left(S U\left(\hat{R}_{1}, x\right)\right) \text { by } s d-E \\
& \geq f\left(\tilde{R}_{N^{1}(\bar{R})}, \bar{R}_{-N^{1}(\bar{R})}\right)\left(S U\left(\hat{R}_{1}, x\right)\right) \text { by } s d-S P \text { and Fact } 2 \\
& =f\left(\tilde{R}_{N^{1}(\bar{R})}, \bar{R}_{-N^{1}(\bar{R})}\right)([\hat{p}, x[) \text { by } s d-E \\
& =f\left(\tilde{R}_{N^{1}(\bar{R})}, \bar{R}_{-N^{1}(\bar{R})}\right)\left(\left[x^{0}, x[) \text { by } s d-E .\right.\right.
\end{aligned}
$$

Thus, $f\left(\tilde{R}_{N^{1}(\bar{R})}, \bar{R}_{-N^{1}(\bar{R})}\right)\left(\left[x^{0}, x[)=f\left(\hat{R}_{1}, \tilde{R}_{N^{1}(\bar{R}) \backslash\{1\}}, \bar{R}_{-N^{1}(\bar{R})}\right)\left(\left[x^{0}, x[)\right.\right.\right.\right.$. Similarly, applying the same argument to the profile $\left(\hat{R}_{1}, \tilde{R}_{N^{1}(\bar{R}) \backslash\{1\}}, \bar{R}_{-N^{1}(\bar{R})}\right)$, we obtain $f\left(\hat{R}_{1}, \tilde{R}_{N^{1}(\bar{R}) \backslash\{1\}}, \bar{R}_{-N^{1}(\bar{R})}\right)\left(\left[x^{0}, x[)=f\left(\hat{R}_{1,2}, \tilde{R}_{N^{1}(\bar{R}) \backslash\{1,2\}}, \bar{R}_{-N^{1}(\bar{R})}\right)\left(\left[x^{0}, x[)\right.\right.\right.\right.$. Repeating this argument for agents $j=3, \ldots, \bar{n}^{k}$, we have

$$
f\left(\tilde{R}_{N^{1}(\bar{R})}, \bar{R}_{-N^{1}(\bar{R})}\right)\left(\left[x^{0}, x[)=f\left(\hat{R}_{\bar{N}}, \tilde{R}_{N^{1}(\bar{R}) \backslash \bar{N}}, \bar{R}_{-N^{1}(\bar{R})}\right)\left(\left[x^{0}, x[) .\right.\right.\right.\right.
$$

If $N^{1}(\bar{R}) \backslash \bar{N}=\emptyset$, then Step 2 follows from (4) and (5). Thus, we assume that $N^{1}(\bar{R}) \backslash \bar{N} \neq \emptyset$. Consider the profile $\left(\hat{R}_{\bar{N}}, \bar{R}_{-\bar{N}}\right)$. Then, $p^{1}\left(\hat{R}_{\bar{N}}, \bar{R}_{-\bar{N}}\right)=p^{1}(\bar{R})=x^{0}$, and $x^{k-1} \leq \hat{p}=p^{2}\left(\hat{R}_{\bar{N}}, \bar{R}_{-\bar{N}}\right)$. It follows from Step 1 that

$$
f\left(\hat{R}_{\bar{N}}, \bar{R}_{-\bar{N}}\right)\left(\left[x^{0}, x[)=f\left(\hat{R}_{\bar{N}}, \tilde{R}_{N^{1}(\bar{R}) \backslash \bar{N}}, \bar{R}_{-N^{1}(\bar{R})}\right)\left(\left[x^{0}, x[) .\right.\right.\right.\right.
$$


Thus, from (4), (5), and (6),

$$
f(\bar{R})\left(\left[x^{0}, x[)=f\left(\hat{R}_{\bar{N}}, \bar{R}_{-\bar{N}}\right)\left(\left[x^{0}, x[) .\right.\right.\right.\right.
$$

Finally, we complete the proof of Lemma 1. Note that (a) $p^{1}(R)=x^{0}$, (b) for each $i \in \bar{N}, p\left(\bar{R}_{i}\right)=\bar{p}$, (c) $\bar{p} \leq p^{2}(R)$, and (d) $x^{K-1}<\bar{p} \leq x^{K}$. Then, it follows from Step 2 that $f(R)\left(\left[x^{0}, x[)=f\left(\bar{R}_{\bar{N}}, R_{-\bar{N}}\right)\left(\left[x^{0}, x[)\right.\right.\right.\right.$.

Remark 5. If the strict upper contour set $S U\left(R_{i}, x\right)$ is replaced by the upper contour set $U\left(R_{i}, x\right)$ in the proof of Lemma 1 , then the result of Lemma 1 also holds for the closed interval, that is, we obtain $f(R)\left(\left[p^{1}(R), x\right]\right)=f\left(\bar{R}_{\bar{N}}, R_{-\bar{N}}\right)\left(\left[p^{1}(R), x\right]\right)$.

Remark 6. The following statements also hold by applying the argument in the proof of Lemma 1 symmetrically. Let $f$ be an sd-strategy-proof and onto rule on $\mathcal{R}^{N}$. Let $R \in \mathcal{R}^{N}, \bar{N} \subseteq N^{\bar{n}}(R), \bar{R}_{\bar{N}} \in \mathcal{R}^{\bar{N}}$, and $x, \bar{p} \in Z$ be such that (1-1*) for each $i \in \bar{N}, p\left(\bar{R}_{i}\right)=\bar{p}$, (1-2*) $p^{\bar{n}-1}(R) \leq \bar{p}<p^{\bar{n}}(R)$, and (1-3*) $x<\bar{p}$. Then, $\left.\left.\left.\left.f(R)(] x, p^{\bar{n}}(R)\right]\right)=f\left(\bar{R}_{\bar{N}}, R_{-\bar{N}}\right)(] x, p^{\bar{n}}(R)\right]\right)$. Similarly to Remark 5 , we also obtain $f(R)\left(\left[x, p^{\bar{n}}(R)\right]\right)=f\left(\bar{R}_{\bar{N}}, R_{-\bar{N}}\right)\left(\left[x, p^{\bar{n}}(R)\right]\right)$.

Lemma 2. Let $f$ be an sd-strategy-proof and onto rule on $\mathcal{R}^{N}$. For each $R \in \mathcal{R}^{N}$, each $k \in\{1, \ldots, \bar{n}(R)-1\}$, each $\bar{N} \subseteq \bigcup_{h=1}^{k} N^{h}(R)$, each $\bar{R}_{\bar{N}} \in \mathcal{R}^{\bar{N}}$, and each $\bar{p}, x \in Z$, if (2-1) for each $i \in \bar{N}, p\left(\bar{R}_{i}\right)=\bar{p}$, (2-2) $p^{k}(R)<\bar{p} \leq p^{k+1}(R)$, and (2-3) $\bar{p}<x$, then, $f(R)\left(\left[p^{1}(R), x[)=f\left(\bar{R}_{\bar{N}}, R_{-\bar{N}}\right)\left(\left[p^{1}(R), x[)\right.\right.\right.\right.$.

Proof of Lemma 2. Let $R \in \mathcal{R}^{N}, k \in\{1, \ldots, \bar{n}(R)-1\}, \bar{N} \subseteq \bigcup_{h=1}^{k} N^{h}(R)$, $\bar{R}_{\bar{N}} \in \mathcal{R}^{\bar{N}}$, and $\bar{p}, x \in Z$ be such that $(2-1)$ for each $i \in \bar{N}, p\left(\bar{R}_{i}\right)=\bar{p},(2-2) p^{k}(R)<$ $\bar{p} \leq p^{k+1}(R)$, and $(2-3) \bar{p}<x$. Without loss of generality, let $\bar{N} \equiv\{1, \ldots,|\bar{N}|\} .{ }^{33}$ Let $x^{0} \equiv p^{1}(R)$. We prove Lemma 2 by induction on $k$. Let $k=1$. Then, the desired conclusion follows from Lemma 1 . Thus, we assume that $k \geq 2$. As the induction hypothesis, we assume that

B: For each $\tilde{R} \in \mathcal{R}^{N}$, each $\hat{k} \in\{1, \ldots, k-1\}$, each $N^{\prime} \subseteq \bigcup_{h=1}^{\hat{k}} N^{h}(\tilde{R})$, each $\hat{R}_{N^{\prime}} \in \mathcal{R}^{N^{\prime}}$, and each $\hat{p}, \hat{x} \in Z$, if (2-1b) for each $i \in N^{\prime}, p\left(\hat{R}_{i}\right)=\hat{p}$, (2-2b) $p^{\hat{k}}(\tilde{R})<$ $\hat{p} \leq p^{\hat{k}+1}(\tilde{R})$, and (2-3b) $\hat{p}<\hat{x}$, then, $f(\tilde{R})\left(\left[p^{1}(\tilde{R}), \hat{x}[)=f\left(\hat{R}_{N^{\prime}}, \tilde{R}_{-N^{\prime}}\right)\left(\left[p^{1}(\tilde{R}), \hat{x}[)\right.\right.\right.\right.$.

Let $\bar{N}^{k-1} \equiv \bigcup_{h=1}^{k-1} N^{h}(R)$. Let $R_{\bar{N}^{k-1}}^{(k)} \in \mathcal{R}^{\bar{N}^{k-1}}$ be such that for each $i \in \bar{N}^{k-1}$, $p\left(R_{i}^{(k)}\right) \equiv p^{k}(R)$. Then, by induction hypothesis (B),

$$
f(R)\left(\left[x^{0}, x[)=f\left(R_{\bar{N}^{k-1}}^{(k)}, R_{-\bar{N}^{k-1}}\right)\left(\left[x^{0}, x[) .\right.\right.\right.\right.
$$

Let $\bar{N}^{k} \equiv \bigcup_{h=1}^{k} N^{h}(R)$. Let $(\hat{N}, \tilde{N})$ be a partition of $\bar{N}$ such that $\hat{N} \cup \tilde{N}=\bar{N}$, $\hat{N} \cap \tilde{N}=\emptyset, \hat{N} \subset \bar{N}^{k-1}$, and $\tilde{N} \subset N^{k}(R)$. Note that for each $i \in \bar{N}, i \in N^{k}(R)$ or $i \in \bar{N}^{k-1}$.

Let $R^{\prime} \equiv\left(R_{\bar{N}^{k-1}}^{(k)}, R_{-\bar{N}^{k-1}}\right)$. Next, we replace the preference $R_{i}^{\prime}$ of agent $i \in \bar{N}$

${ }^{33}|A|$ denotes the cardinality of the set $A$. 
with the preference $\bar{R}_{i}$, inductively. Then, by $p^{1}\left(R^{\prime}\right)=p^{k}(R)$ and $\bar{N} \subset N^{1}\left(R^{\prime}\right)$,

$$
\begin{aligned}
f\left(R^{\prime}\right)\left(\left[x^{0}, x[)\right.\right. & =f\left(R^{\prime}\right)\left(\left[p^{k}(R), x[) \text { by } s d-E\right.\right. \\
& =f\left(\bar{R}_{\bar{N}}, R_{-\bar{N}}^{\prime}\right)\left(\left[p^{k}(R), x[) \text { by Lemma } 1\right.\right. \\
& =f\left(\bar{R}_{\bar{N}}, R_{-\bar{N}}^{\prime}\right)\left(\left[x^{0}, x[) \text { by } s d-E .\right.\right.
\end{aligned}
$$

Since $R^{\prime} \equiv\left(R_{\bar{N}^{k-1}}^{(k)}, R_{-\bar{N}^{k-1}}\right)$

$$
f\left(R_{\bar{N}^{k-1}}^{(k)}, R_{-\bar{N}^{k-1}}\right)\left(\left[x^{0}, x[)=f\left(\bar{R}_{\bar{N}}, R_{\bar{N}^{k-1} \backslash \hat{N}}^{(k)}, R_{N^{k}(R) \backslash \tilde{N}}, R_{-\bar{N}^{k}}\right)\left(\left[x^{0}, x[) .\right.\right.\right.\right.
$$

If $\bar{N}^{k-1} \backslash \hat{N}=\emptyset$, then Lemma 2 follows from (7) and (8). Thus, we assume that $\bar{N}^{k-1} \backslash \hat{N} \neq \emptyset$.

Next, consider the profile $\left(\bar{R}_{\bar{N}}, R_{-\bar{N}}\right)$. Note that $p^{k}(R) \leq p^{k}\left(\bar{R}_{\bar{N}}, R_{-\bar{N}}\right)$. Then, by induction hypothesis (B), ${ }^{34}$

$$
f\left(\bar{R}_{\bar{N}}, R_{-\bar{N}}\right)\left(\left[x^{0}, x[)=f\left(\bar{R}_{\bar{N}}, R_{\bar{N}^{k-1} \backslash \hat{N}}^{(k)}, R_{N^{k}(R) \backslash \tilde{N}}, R_{-\bar{N}^{k}}\right)\left(\left[x^{0}, x[) .\right.\right.\right.\right.
$$

Thus, from (7), (8), and (9),

$$
f(R)\left(\left[p^{1}(R), x[)=f\left(\bar{R}_{\bar{N}}, R_{-\bar{N}}\right)\left(\left[p^{1}(R), x[) .\right.\right.\right.\right.
$$

Remark 7. If the result of Remark 5 is applied to the proof of Lemma 2 instead of Lemma 1, the result of Lemma 2 also holds for the closed interval, that is, we obtain $f(R)\left(\left[p^{1}(R), x\right]\right)=f\left(\bar{R}_{\bar{N}}, R_{-\bar{N}}\right)\left(\left[p^{1}(R), x\right]\right)$.

Remark 8. The following statements also hold by applying the argument in the proof of Lemma 2 symmetrically. Let $f$ be an sd-strategy-proof and onto rule on $\mathcal{R}^{N}$. For each $R \in \mathcal{R}^{N}$, each $k \in\{2, \ldots, \bar{n}(R)\}$, each $\bar{N} \subseteq \bigcup_{h=k}^{\bar{n}(R)} N^{h}(R)$, each $\bar{R}_{\bar{N}} \in \mathcal{R}^{\bar{N}}$, and each $x, \bar{p} \in Z$, if (2-1*) for each $i \in \bar{N}, p\left(\bar{R}_{i}\right)=\bar{p}$, (2-2*) $p^{k-1}(R) \leq \bar{p}<p^{k}(R)$, and (2-3*) $x<\bar{p}$, then, $\left.\left.\left.\left.f(R)(] x, p^{\bar{n}}(R)\right]\right)=f\left(\bar{R}_{\bar{N}}, R_{-\bar{N}}\right)(] x, p^{\bar{n}}(R)\right]\right)$. Similarly to Remark 7 , we also obtain $f(R)\left(\left[x, p^{\bar{n}}(R)\right]\right)=f\left(\bar{R}_{\bar{N}}, R_{-\bar{N}}\right)\left(\left[x, p^{\bar{n}}(R)\right]\right)$.

Proof of Proposition 1. Let $R \in \mathcal{R}^{N}, i \in N$, and $\hat{R}_{i} \in \mathcal{R}$. Let $[x, y[\subset Z$ be such that $\left[x, y\left[\cap E\left(R_{i}, \hat{R}_{i}\right)=\emptyset\right.\right.$. We show that $f(R)\left(\left[x, y[)=f\left(\hat{R}_{i}, R_{-i}\right)\left(\left[x, y[){ }^{35}\right.\right.\right.\right.$ If $p\left(R_{i}\right)=p\left(\hat{R}_{i}\right)$, then, by condition (ii) of the minimally rich domain in Definition 1 , $R_{i}=\hat{R}_{i}$. Thus, we consider the case where $p\left(R_{i}\right) \neq p\left(\hat{R}_{i}\right)$. Assume that $p\left(R_{i}\right)<$ $p\left(\hat{R}_{i}\right)$. The opposite case can be treated symmetrically.

Case 1. $p\left(\hat{R}_{i}\right)<x$.

If $p\left(\hat{R}_{i}\right) \geq p^{\bar{n}(R)}(R)$, then, by sd-efficiency, $f(R)\left(\left[x, y[)=0=f\left(\hat{R}_{i}, R_{-i}\right)([x, y[)\right.\right.$. Thus, assume that $p\left(\hat{R}_{i}\right)<p^{\bar{n}(R)}(R)$. Then, there is $k \in\{1, \ldots, \bar{n}(R)-1\}$ such that $p^{k}(R)<p\left(\hat{R}_{i}\right) \leq p^{k+1}(R)$. Since $p\left(R_{i}\right)<p\left(\hat{R}_{i}\right), i \in \bigcup_{h=1}^{k} N^{h}(R)$. Then, it follows

\footnotetext{
${ }^{34}$ By letting $\tilde{R} \equiv\left(\bar{R}_{\bar{N}}, R_{-\bar{N}}\right), N^{\prime} \equiv \bar{N}^{k-1} \backslash \hat{N}, \hat{p} \equiv p^{k}(R)$, and $\hat{x} \equiv x$, all the assumptions (2-1b), $(2-2 \mathrm{~b})$, and $(2-3 \mathrm{~b})$ of induction hypothesis $(\mathrm{B})$ are satisfied.

${ }^{35}$ To show the equivalence of two distributions $g$ and $h$ over $Z$, it is sufficient to prove that for each $[x, y[\subset Z, g([x, y[)=h([x, y[)$. See Ehlers et al. (2002, Remark A.3. in the Appendix).
} 
from Lemma 2 that $f(R)\left(\left[p^{1}(R), x[)=f\left(\hat{R}_{i}, R_{-i}\right)\left(\left[p^{1}(R), x[)\right.\right.\right.\right.$. Similarly, by $x<y$, $f(R)\left(\left[p^{1}(R), y[)=f\left(\hat{R}_{i}, R_{-i}\right)\left(\left[p^{1}(R), y[)\right.\right.\right.\right.$. Thus,

$$
\begin{aligned}
f(R)([x, y[) & =f(R)\left(\left[p^{1}(R), y[)-f(R)\left(\left[p^{1}(R), x[)\right.\right.\right.\right. \\
& =f\left(\hat{R}_{i}, R_{-i}\right)\left(\left[p^{1}(R), y[)-f\left(\hat{R}_{i}, R_{-i}\right)\left(\left[p^{1}(R), x[)\right.\right.\right.\right. \\
& =f\left(\hat{R}_{i}, R_{-i}\right)([x, y[) .
\end{aligned}
$$

Case 2. $y \leq p\left(R_{i}\right)$.

If $p\left(R_{i}\right)=p^{1}(R)$, then, by sd-efficiency, $f(R)\left(\left[x, y[)=0=f\left(\hat{R}_{i}, R_{-i}\right)([x, y[)\right.\right.$. Thus, assume that $p^{1}(R)<p\left(R_{i}\right)$. Consider the profile $\hat{R} \equiv\left(\hat{R}_{i}, R_{-i}\right)$. Then, there is $\bar{k} \in\{2, \ldots, \bar{n}(\hat{R})\}$ such that $p^{\bar{k}-1}(\hat{R}) \leq p\left(R_{i}\right)<p^{\bar{k}}(\hat{R})$. Since $p\left(R_{i}\right)<p\left(\hat{R}_{i}\right)$, $i \in \bigcup_{h=\bar{k}}^{\bar{n}(\hat{R})} N^{h}(\hat{R})$. Let $\hat{p} \equiv p^{\bar{n}(\hat{R})}(\hat{R})$. First, we assume that $y<p\left(R_{i}\right)$. Then, it follows from Remark 8 that $f(R)([y, \hat{p}])=f\left(\hat{R}_{i}, R_{-i}\right)([y, \hat{p}])$. Similarly, by $x<y$, $f(R)([x, \hat{p}])=f\left(\hat{R}_{i}, R_{-i}\right)([x, \hat{p}])$. Thus, $f(R)\left(\left[x, y[)=f\left(\hat{R}_{i}, R_{-i}\right)([x, y[)\right.\right.$. Next, we assume that $y=p\left(R_{i}\right)$. Let $\left\{y_{k}\right\}_{k \in \mathbb{N}}$ be a sequence in $Z$ such that for each $k \in \mathbb{N}$, $y_{k}<y,\left[x, y_{k}\left[\subseteq\left[x, y_{k+1}\left[\right.\right.\right.\right.$, and $\bigcup_{k \in \mathbb{N}}\left[x, y_{k}\left[=\left[x, y\left[{ }^{36}\right.\right.\right.\right.$ Then, for each $k \in \mathbb{N}$, $y_{k}<p\left(R_{i}\right)$. Thus, by applying the above argument again, for each $k \in \mathbb{N}$, we have $f(R)\left(\left[x, y_{k}[)=f\left(\hat{R}_{i}, R_{-i}\right)\left(\left[x, y_{k}[)\right.\right.\right.\right.$. Since distributions are continuous from below, ${ }^{37}$ $f(R)\left(\left[x, y[)=f\left(\hat{R}_{i}, R_{-i}\right)([x, y[)\right.\right.$.

Proof of Theorem 1. It is easy to show that each probabilistic generalized median rule is sd-strategy-proof and onto. The proof of the uniqueness part of Theorem 1 is similar to that of Ehlers et al. (2002), but we provide it for completeness. Let $f$ be an sd-strategy-proof and onto rule defined on $\mathcal{R}^{N}$.

Step 1. Construction of a collection $\left(D_{S}\right)_{S \in 2^{N}}$ of probability distributions.

For each $i \in N$, let $R_{i}^{z}, R_{i}^{\bar{z}} \in \mathcal{R}$ be such that $p\left(R_{i}^{z}\right) \equiv \underline{z}$ and $p\left(R_{i}^{\bar{z}}\right) \equiv \bar{z}$. For each $S \in 2^{N}$, let $D_{S} \equiv f\left(R_{S}^{z}, R_{-S}^{\bar{z}}\right)$.

Step 2. The collection $D \equiv\left(D_{S}\right)_{S \in 2^{N}}$ satisfies the following properties:

(i) $D_{\emptyset}(\{\bar{z}\})=1$, (ii) $D_{N}(\{\underline{z}\})=1$, and (iii) for each $S, T \in 2^{N}$ and each $x \in Z$, $D_{S \cup T}([\underline{z}, x])-D_{S}([\underline{z}, x[) \geq 0$.

By unanimity, (i) $D_{\emptyset}(\{\bar{z}\}) \equiv f\left(R_{N}^{\bar{z}}\right)(\{\bar{z}\})=1$, and (ii) $D_{N}(\{\underline{z}\}) \equiv f\left(R_{N}^{\underline{z}}\right)(\{\underline{z}\})=$ 1. We show property (iii) of Step 2. Let $S, T \in 2^{N}$ and $x \in Z$. Without loss of generality, assume that $S \cap T \neq \emptyset$ and $T \equiv\{1,2, \ldots, \hat{t}\}$. Then,

$$
\begin{aligned}
D_{S \cup\{1\}}([\underline{z}, x]) & \equiv f\left(R_{S}^{z}, R_{1}^{z}, R_{-(S \cup\{1\})}^{\bar{z}}\right)([\underline{z}, x]) \\
& \geq f\left(R_{S}^{z}, R_{1}^{z}, R_{-(S \cup\{1\})}^{\bar{z}}\right)([\underline{z}, x[) \\
& =f\left(R_{\bar{S}}^{z}, R_{1}^{z}, R_{-(S \cup\{1\})}^{\bar{z}}\right)\left(S U\left(R_{1}^{z}, x\right)\right) \\
& \geq f\left(R_{\bar{S}}^{\underline{z}}, R_{-S}^{\bar{z}}\right)\left(S U\left(R_{1}^{z}, x\right)\right) \text { by } s d-S P \text { and Fact } 2 \\
& =f\left(R_{S}^{z}, R_{-S}^{\bar{z}}\right)([\underline{z}, x[) \\
& =D_{S}([\underline{z}, x[) .
\end{aligned}
$$

\footnotetext{
${ }^{36}$ For each $k \in \mathbb{N}$, set $y_{k} \equiv y-\frac{y-x}{2 k}$. Then, the sequence $\left\{y_{k}\right\}_{k \in \mathbb{N}}$ satisfies these properties.

${ }^{37}$ A probability distribution $g$ is continuous from below if for each sequence $\left\{A_{k}\right\}_{k \in \mathbb{N}}$ such that for each $k \in \mathbb{N}, A_{k} \subseteq A_{k+1}$ and $\bigcup_{k \in \mathbb{N}} A_{k}=A, g(A)=\lim _{k \rightarrow \infty} g\left(A_{k}\right)$. See Ehlers et al. (2002, Theorem A.1. in the Appendix).
} 
Repeating this argument for agents $j=2, \ldots, \hat{t}$, we get $D_{S \cup T}([\underline{z}, x])-D_{S}([\underline{z}, x[) \geq 0$.

Step 3. For each $R \in \mathcal{R}^{N}, f(R)=g m^{D}(R){ }^{38}$

Let $R \in \mathcal{R}^{N}$. By sd-efficiency, $f(R)\left(\left[\underline{z}, p^{1}(R)[)=0=D_{\emptyset}\left(\left[\underline{z}, p^{1}(R)[)\right.\right.\right.\right.$, and $\left.\left.\left.\left.f(R)(] p^{\bar{n}}(R), \bar{z}\right]\right)=0=D_{N}(] p^{\bar{n}}(R), \bar{z}\right]\right)$. Let $\ell \in\{1, \ldots, \bar{n}(R)-1\}$.

First, we show that $f(R)(] p^{\ell}(R), p^{\ell+1}(R)[)=g m^{D}(R)(] p^{\ell}(R), p^{\ell+1}(R)[)$. By uncompromisingness,

$$
f(R)(] p^{\ell}(R), p^{\ell+1}(R)[)=f\left(R_{S_{\ell}}^{z}, R_{-S_{\ell}}^{\bar{z}}\right)(] p^{\ell}(R), p^{\ell+1}(R)[) \equiv D_{S_{\ell}}(] p^{\ell}(R), p^{\ell+1}(R)[) .
$$

Let $\ell \in\{1, \ldots, \bar{n}(R)\}$. Next, we show that $f(R)\left(\left\{p^{\ell}(R)\right\}\right)=g m^{D}(R)\left(\left\{p^{\ell}(R)\right\}\right)$. First,

$$
\begin{aligned}
\left.\left.f(R)(] p^{\ell}(R), \bar{z}\right]\right) & \left.\left.=f\left(R_{S_{\ell}}^{z}, R_{-S_{\ell}}\right)(] p^{\ell}(R), \bar{z}\right]\right) \text { by uncompromisingness } \\
& =1-f\left(R_{\bar{S}_{\ell}}^{z}, R_{-S_{\ell}}\right)\left(\left[\underline{z}, p^{\ell}(R)\right]\right) \\
& =1-f\left(R_{S_{\ell}}^{\bar{z}}, R_{-S_{\ell}}^{\bar{z}}\right)\left(\left[\underline{z}, p^{\ell}(R)\right]\right) \text { by uncompromisingness } \\
& \left.\left.=f\left(R_{S_{\ell}}^{z}, R_{-S_{\ell}}^{\bar{z}}\right)(] p^{\ell}(R), \bar{z}\right]\right) .
\end{aligned}
$$

Similarly,

$$
\begin{aligned}
f(R)\left(\left[\underline{z}, p^{\ell}(R)[)\right.\right. & =f\left(R_{S_{\ell-1}}, R_{-S_{\ell-1}}^{\bar{z}}\right)\left(\left[\underline{z}, p^{\ell}(R)[)\right.\right. \text { by uncompromisingness } \\
& =1-f\left(R_{S_{\ell-1}}, R_{-S_{\ell-1}}^{\bar{z}}\right)\left(\left[p^{\ell}(R), \bar{z}\right]\right) \\
& =1-f\left(R_{S_{\ell-1}}^{z_{\ell-1}}, R_{-S_{\ell-1}}^{\bar{z}}\right)\left(\left[p^{\ell}(R), \bar{z}\right]\right) \text { by uncompromisingness } \\
& =f\left(R_{S_{\ell-1}}^{z}, R_{-S_{\ell-1}}^{\bar{z}}\right)\left(\left[\underline{z}, p^{\ell}(R)[) .\right.\right.
\end{aligned}
$$

Thus, we have

$$
\begin{aligned}
f(R)\left(\left\{p^{\ell}(R)\right\}\right) & \left.\left.=1-f(R)(] p^{\ell}(R), \bar{z}\right]\right)-f(R)\left(\left[\underline{z}, p^{\ell}(R)[)\right.\right. \\
& \left.\left.=1-f\left(R_{S_{\ell}}^{z}, R_{-S_{\ell}}^{\bar{z}}\right)(] p^{\ell}(R), \bar{z}\right]\right)-f\left(R_{S_{\ell-1}}^{z}, R_{-S_{\ell-1}}^{\bar{z}}\right)\left(\left[\underline{z}, p^{\ell}(R)[)\right.\right. \\
& =f\left(R_{S_{\ell}}^{\underline{z}}, R_{-S_{\ell}}^{\bar{z}}\right)\left(\left[\underline{z}, p^{\ell}(R)\right]\right)-f\left(R_{S_{\ell-1}}^{\underline{z}}, R_{-S_{\ell-1}}^{\bar{z}}\right)\left(\left[\underline{z}, p^{\ell}(R)[)\right.\right. \\
& \equiv D_{S_{\ell}}\left(\left[\underline{z}, p^{\ell}(R)\right]\right)-D_{S_{\ell-1}}\left(\left[\underline{z}, p^{\ell}(R)[) .\right.\right.
\end{aligned}
$$

\subsection{Proof of Theorem 2.}

Next, we prove Theorem 2. The proof structure is similar to that of Berga and Serizawa (2000), and we borrow some of their techniques. However, the class of probabilistic rules is much larger than that of deterministic rules, and the notion of sd-strategy-proofness is different from strategy-proofness for deterministic rules.

\footnotetext{
${ }^{38}$ Recall that the distribution of $g m^{D}$ is equal to the distribution $D_{\emptyset}$ on the interval $\left[\underline{z}, p^{1}(R)[\right.$, $D_{N}$ on the interval $\left.] p^{\bar{n}}(R), \bar{z}\right]$, and for each $\ell \in\{1, \ldots, \bar{n}-1\}, D_{S_{\ell}}$ on the interval $] p^{\ell}(R), p^{\ell+1}(R)[$. Further, for each $\ell \in\{1, \ldots, \bar{n}\}$, the probability assigned to the $\ell$-th peak, $g m^{D}(R)\left(\left\{p^{\ell}(R)\right\}\right)$, is equal to $D_{S_{\ell}}\left(\left[\underline{z}, p^{\ell}(R)\right]\right)-D_{S_{\ell-1}}\left(\left[\underline{z}, p^{\ell}(R)[)\right.\right.$.
} 
Furthermore, in the proof of Theorem 2, we use our characterization result of $s d$ strategy-proof and onto rules on a minimally rich domain (Theorem 1). Thus, we cannot directly apply their proofs.

Lemma 3 (Berga and Serizawa, 2000, Lemma 3). Let $g$ be a continuous realvalued function defined on $Z$ such that for some $x, y, z \in Z, x<y<z, g(x)>g(y)$ and $g(z)>g(y)$. Then,

(a) there exists a triple $a, b, r \in Z$, and for any $\varepsilon>0$, there exists $c$ such that $c \in] r, r+\varepsilon[$ which satisfy the following four conditions: (i) $x \leq a<b<r<c \leq z$, (ii) $g(a)=g(r)$, (iii) if $\left.x^{\prime} \in\right] a, r\left[, g(a)>g\left(x^{\prime}\right)\right.$, and (iv) if $x^{\prime} \in\left[a, c\left[, g(c)>g\left(x^{\prime}\right)\right.\right.$, and

(b) there exists a triple $r^{\prime}, b^{\prime}, c^{\prime} \in Z$, and for any $\varepsilon>0$, there exists $a^{\prime}$ such that $\left.a^{\prime} \in\right] r^{\prime}-\varepsilon, r^{\prime}\left[\right.$ which satisfy the following four conditions: (i) $x \leq a^{\prime}<r^{\prime}<b^{\prime}<$ $c^{\prime} \leq z$, (ii) $g\left(r^{\prime}\right)=g\left(c^{\prime}\right)$, (iii) if $\left.x^{\prime} \in\right] r^{\prime}, c^{\prime}\left[, g\left(c^{\prime}\right)>g\left(x^{\prime}\right)\right.$, and (iv) if $\left.\left.x^{\prime} \in\right] a^{\prime}, c^{\prime}\right]$, $g\left(a^{\prime}\right)>g\left(x^{\prime}\right)$.

Let $\underline{\mathcal{R}} \in \mathcal{R}^{M R}$. Let $\mathcal{R} \subset \mathcal{P}$ be a domain that includes $\underline{\mathcal{R}}$.

Lemma 4. Let $f$ be a rule on $\mathcal{R}^{N}$ satisfying sd-strategy-proofness, ontoness, and the no-vetoer condition. Let $z, z^{\prime} \in Z$ with $z \leq z^{\prime}, i \in N, R_{i}^{0} \in \mathcal{R}$, and $R_{-i} \in$ $\underline{\mathcal{R}}^{N \backslash\{i\}}$ be such that for each $j \in N \backslash\{i\}$, either $p\left(R_{j}\right)=z$ or $p\left(R_{j}\right)=z^{\prime}$. Then, $f\left(R_{i}^{0}, R_{-i}\right)\left(\left[z, z^{\prime}\right]\right)=1$.

Proof of Lemma 4. By contradiction, suppose that $f\left(R_{i}^{0}, R_{-i}\right)\left(\left[z, z^{\prime}\right]\right)<1$. Without loss of generality, assume that $f\left(R_{i}^{0}, R_{-i}\right)\left(\left[\underline{z}, z[)>0\right.\right.$. Let $R_{i}^{\underline{z}} \in \underline{\mathcal{R}}$ be such that $p\left(R_{i}^{z}\right) \equiv \underline{z}$. Then,

$$
\begin{aligned}
f\left(R_{i}^{z}, R_{-i}\right)([\underline{z}, z[) & =f\left(R_{i}^{z}, R_{-i}\right)\left(S U\left(R_{i}^{z}, z\right)\right) \\
& \geq f\left(R_{i}^{0}, R_{-i}\right)\left(S U\left(R_{i}^{z}, z\right)\right) \text { by } s d-S P \text { and Fact } 2 \\
& =f\left(R_{i}^{0}, R_{-i}\right)([\underline{z}, z[) \\
& >0 .
\end{aligned}
$$

For each $j \in N \backslash\{i\}$, let $R_{j}^{\bar{z}} \in \underline{\mathcal{R}}$ be such that $p\left(R_{j}^{\bar{z}}\right) \equiv \bar{z}$. By Proposition 1 , any $s d$ strategy-proof and onto probabilistic rule $f$ defined on $\underline{\mathcal{R}}^{N}$ is uncompromising, and so, $f\left(R_{i}^{\underline{z}}, R_{-i}^{\bar{z}}\right)\left(\left[\underline{z}, z[)=f\left(R_{i}^{\underline{z}}, R_{-i}\right)\left(\left[\underline{z}, z[)>0\right.\right.\right.\right.$. Thus, $f\left(R_{i}^{\underline{z}}, R_{-i}^{\bar{z}}\right)(\{\bar{z}\}) \neq 1$, which contradicts the no-vetoer condition.

Lemma 5. Let $f$ be an sd-strategy-proof rule on $\mathcal{R}^{N}$. Let $a, b \in Z$ with $a \neq b$ and $X \equiv[a, b[,[a, b]] b, a$,$] , or [b, a]$. Let $R_{0}^{a} \in \underline{\mathcal{R}}$ be such that $p\left(R_{0}^{a}\right)=a$. Let $\bar{N} \subset N$, $R_{\bar{N}}^{0} \in \mathcal{R}^{\bar{N}}$, and $R_{\bar{N}}^{a} \in \underline{\mathcal{R}}^{\bar{N}}$ be such that for each $i \in \bar{N}, R_{i}^{a}=R_{0}^{a}$. Let $R_{-\bar{N}} \in \mathcal{R}^{N \backslash \bar{N}}$. Assume that $f\left(R_{\bar{N}}^{0}, R_{-\bar{N}}\right)(X)>0$. Then,

(a) if $X=[a, b[$ or $] b, a]$, then $f\left(R_{\bar{N}}^{a}, R_{-\bar{N}}\right)\left(S U\left(R_{0}^{a}, b\right)\right)>0$, and

(b) if $X=[a, b]$ or $[b, a]$, then $f\left(R_{\bar{N}}^{a}, R_{-\bar{N}}\right)\left(U\left(R_{0}^{a}, b\right)\right)>0$.

Proof of Lemma 5. We prove (a) of Lemma 5 . Let $X \equiv[a, b[$. (The same argument applies to the other case.) Without loss of generality, let $\bar{N} \equiv\{1,2, \ldots,|\bar{N}|\}$. 
Note that $\left[a, b\left[\subset S U\left(R_{0}^{a}, b\right)\right.\right.$. Then,

$$
\begin{aligned}
f\left(R_{1}^{a}, R_{\bar{N} \backslash\{1\}}^{0}, R_{-\bar{N}}\right)\left(S U\left(R_{1}^{a}, b\right)\right) & \geq f\left(R_{\bar{N}}^{0}, R_{-\bar{N}}\right)\left(S U\left(R_{1}^{a}, b\right)\right) \text { by } s d-S P \text { and Fact } 2 \\
& \geq f\left(R_{\bar{N}}^{0}, R_{-\bar{N}}\right)\left(\left[a , b [ ) \text { by } \left[a, b\left[\subset S U\left(R_{0}^{a}, b\right)\right.\right.\right.\right. \\
& >0 .
\end{aligned}
$$

Since $R_{1}^{a}=R_{0}^{a}=R_{2}^{a}$,

$$
\begin{aligned}
f\left(R_{1,2}^{a}, R_{\bar{N} \backslash\{1,2\}}^{0}, R_{-\bar{N}}\right) & \left.S U\left(R_{2}^{a}, b\right)\right) \\
\geq & f\left(R_{1}^{a}, R_{\bar{N} \backslash\{1\}}^{0}, R_{-\bar{N}}\right)\left(S U\left(R_{2}^{a}, b\right)\right) \text { by sd-SP and Fact } 2 \\
& =f\left(R_{1}^{a}, R_{\bar{N} \backslash\{1\}}^{0}, R_{-\bar{N}}\right)\left(S U\left(R_{1}^{a}, b\right)\right) \text { by } R_{1}^{a}=R_{2}^{a} \\
& >0 .
\end{aligned}
$$

Repeating this argument for agents $j=3, \ldots,|\bar{N}|, f\left(R_{\bar{N}}^{a}, R_{-\bar{N}}\right)\left(S U\left(R_{0}^{a}, b\right)\right)>0$.

By replacing the strict upper contour set $S U\left(R_{0}^{a}, b\right)$ by the upper contour set $U\left(R_{0}^{a}, b\right)$ in the proof of (a), we can also prove (b) of Lemma 5.

Proof of Theorem 2. First, we show condition (i) in the definition of maximal domain. ${ }^{39}$ For each $R \in\left(\mathcal{R}^{\text {vex }}\right)^{N}$ and each $i \in N$, define $\hat{p}\left(R_{i}\right) \equiv \min \{x: x \in$ $\left.p\left(R_{i}\right)\right\}$. Let $D \equiv\left(D_{S}\right)_{S \in 2^{N}}$ be a collection of probability distributions such that for each $S_{1} \in 2^{N}$ with $\left|S_{1}\right| \leq 1, D_{S_{1}}(\{\bar{z}\})=1$, and for each $S_{n-1} \in 2^{N}$ with $\left|S_{n-1}\right| \geq$ $n-1, D_{S_{n-1}}(\{\underline{z}\})=1$, and for each $S, T \in 2^{N}$, and each $x \in Z, D_{S \cup T}([\underline{z}, x])-$ $D_{S}\left(\left[\underline{z}, x[) \geq 0\right.\right.$. For each $R \in\left(\mathcal{R}^{v e x}\right)^{N}$, define $f(R) \equiv g m^{D}\left(\left(\hat{p}\left(R_{i}\right)\right)_{i \in N}\right)$. Then, the rule $f$ satisfies sd-strategy-proofness, ontoness, and the no-vetoer condition.

Next, we show condition (ii) in the definition of maximal domain. Let $\mathcal{R} \subset \mathcal{P}$ be such that $\underline{\mathcal{R}} \subset \mathcal{R}$. Assume that there is a rule $f$ on $\mathcal{R}$ satisfying the three axioms in Theorem 2. Let $\left.f\right|_{\mathcal{R}}$ be the restriction of $f$ to $\underline{\mathcal{R}}^{N}$. Then, by Theorem 1 , there is $D \in \Delta$ such that for each $R \in \underline{\mathcal{R}}^{N},\left.f\right|_{\mathcal{R}}(R)=g m^{\bar{D}}(p(R))$. We show that $\mathcal{R} \subseteq \mathcal{R}^{\text {vex }}$. By contradiction, suppose that there is a non-convex preference $R^{0} \in \mathcal{R} \backslash \mathcal{R}^{\text {vex }}$. Then, by non-convexity of $R^{0}$, there is a triple $x, y, z \in Z$ such that $x<y<z$ and $x P^{0} y$ and $z P^{0} y$.

We introduce some notations. Let $Z\left(R^{0}\right)$ be the set of triples $(a, b, r) \in Z^{3}$ such that $a I^{0} r, a P^{0} b, \underline{z}^{0} \equiv \min \{a, r\}<b<\max \{a, r\} \equiv \bar{z}^{0}$, and for each $\left.x^{\prime} \in\right] \underline{z}^{0}, \bar{z}^{0}[$, a $P^{0} x^{\prime}$.

Let $(a, r) \in Z^{2}$ with $a \neq r$. Define $\underline{\mathcal{R}}(a, r) \equiv\{\bar{R} \in \underline{\mathcal{R}}: r \bar{P} a$, and $\min \{a, r\}<$ $p(\bar{R})<\max \{a, r\}\}$. Note that, by condition (iii-a) of the minimally rich domain in Definition 1 , for each pair $\left(a^{\prime}, r^{\prime}\right) \in Z^{2}$ with $a^{\prime} \neq r^{\prime}, \underline{\mathcal{R}}\left(a^{\prime}, r^{\prime}\right) \neq \emptyset$.

Let $\bar{R} \in \underline{\mathcal{R}}(a, r)$. Let $E(\bar{R}, a) \equiv\{e \in Z: e \bar{I} a$, and $e \neq a\}$. Let $e(\bar{R}, a) \in Z$ be such that (i) if $E(\bar{R}, a) \neq \emptyset$, then $e(\bar{R}, a) \in E(\bar{R}, a),{ }^{40}$ (ii) if $E(\bar{R}, a)=\emptyset$ and $a<p(\bar{R})$, then $e(\bar{R}, a)=\bar{z}$, and (iii) if $E(\bar{R}, a)=\emptyset$ and $p(\bar{R})<a$, then $e(\bar{R}, a)=\underline{z}$.

Let $C\left(R^{0}, a\right) \equiv\left\{c \in Z\right.$ : for each $\hat{x} \in[\min \{a, c\}, \max \{a, c\}]$ with $\hat{x} \neq c, c P^{0}$ $\hat{x}\}$, and $C\left(R^{0},(a, r), \bar{R}\right) \equiv\left\{c \in Z: c \in C\left(R^{0}, a\right)\right.$ and $\min \{r, e(\bar{R}, a)\}<c<$ $\max \{r, e(\bar{R}, a)\}\}$.

\footnotetext{
${ }^{39}$ Since any deterministic rule is included in the class of probabilistic rules as a special case, condition (i) also follows from the result of Berga and Serizawa (2000).

${ }^{40}$ Note that if $E(\bar{R}, a) \neq \emptyset$, then $E(\bar{R}, a)$ is a singleton.
} 
Given $x \in Z$, we denote by $R^{(x)} \in \underline{\mathcal{R}}$ the preference relation whose peak $p\left(R^{(x)}\right)$ is equal to the alternative $x$.

Claim 1. For each $k \in\{1,2, \ldots, n\}$, each $(a, b, r) \in Z\left(R^{0}\right)$, each $\bar{R} \in \underline{\mathcal{R}}(a, r)$, each $c \in C\left(R^{0},(a, r), \bar{R}\right)$, and each $N^{\prime} \subseteq N$ with $\left|N^{\prime}\right|=k, f\left(R_{N^{\prime}}^{(c)}, R_{-N^{\prime}}^{(a)}\right)(\{c\})=0$.

Proof of Claim 1. Let $k \in\{1,2, \ldots, n\},(a, b, r) \in Z\left(R^{0}\right), \bar{R} \in \underline{\mathcal{R}}(a, r), c \in$ $C\left(R^{0},(a, r), \bar{R}\right)$, and $N^{\prime} \subseteq N$ with $\left|N^{\prime}\right|=k$. Without loss of generality, assume that $a<r<c$. Let $k=1$. Then, the desired conclusion follows from Lemma 4 . Assume that $k \geq 2$. We prove Claim 1 by induction on $k$. Let $k=2$. Without loss of generality, let $N^{\prime} \equiv\{1,2\}$. We show that $f\left(R_{1,2}^{(c)}, R_{-1,2}^{(a)}\right)(\{c\})=0$. By contradiction, suppose that $f\left(R_{1,2}^{(c)}, R_{-1,2}^{(a)}\right)(\{c\})>0$.

By Lemma $4, f\left(R_{1}^{(a)}, \bar{R}_{2}, R_{-1,2}^{(a)}\right)(\{a\})=1$. Then,

$$
\begin{aligned}
f\left(R_{1}^{0}, \bar{R}_{2}, R_{-1,2}^{(a)}\right)\left(U\left(R_{1}^{0}, a\right)\right) & \geq f\left(R_{1}^{(a)}, \bar{R}_{2}, R_{-1,2}^{(a)}\right)\left(U\left(R_{1}^{0}, a\right)\right) \text { by } s d-S P \\
& \geq f\left(R_{1}^{(a)}, \bar{R}_{2}, R_{-1,2}^{(a)}\right)(\{a\}) \text { by }\{a\} \subset U\left(R_{1}^{0}, a\right) \\
& =1 .
\end{aligned}
$$

Also, by Lemma $4, f\left(R_{1}^{0}, \bar{R}_{2}, R_{-1,2}^{(a)}\right)\left(\left[a, p\left(\bar{R}_{2}\right)\right]\right)=1$. Since $] a, r\left[\nsubseteq U\left(R_{1}^{0}, a\right)\right.$, we have $f\left(R_{1}^{0}, \bar{R}_{2}, R_{-1,2}^{(a)}\right)(\{a\})=1$, and so, $f\left(R_{1}^{0}, \bar{R}_{2}, R_{-1,2}^{(a)}\right)\left(U\left(\bar{R}_{2}, c\right)\right)=0$.

However, since $f\left(R_{1,2}^{(c)}, R_{-1,2}^{(a)}\right)(\{c\})>0$,

$$
\begin{aligned}
f\left(R_{1}^{0}, R_{2}^{(c)}, R_{-1,2}^{(a)}\right)\left(U\left(R_{1}^{0}, c\right)\right) & \geq f\left(R_{1}^{(c)}, R_{2}^{(c)}, R_{-1,2}^{(a)}\right)\left(U\left(R_{1}^{0}, c\right)\right) \text { by } s d-S P \\
& \geq f\left(R_{1}^{(c)}, R_{2}^{(c)}, R_{-1,2}^{(a)}\right)(\{c\}) \text { by }\{c\} \subset U\left(R_{1}^{0}, c\right) \\
& >0 .
\end{aligned}
$$

By Lemma 4, $f\left(R_{1}^{0}, R_{2}^{(c)}, R_{-1,2}^{(a)}\right)([a, c])=1$. Then, $f\left(R_{1}^{0}, R_{2}^{(c)}, R_{-1,2}^{(a)}\right)(\{c\})>0$. Thus, $f\left(R_{1}^{0}, R_{2}^{(c)}, R_{-1,2}^{(a)}\right)\left(U\left(\bar{R}_{2}, c\right)\right)>0=f\left(R_{1}^{0}, \bar{R}_{2}, R_{-1,2}^{(a)}\right)\left(U\left(\bar{R}_{2}, c\right)\right)$, which contradicts sd-strategy-proofness. Thus, Claim 1 holds if $k=2$.

Let $k \geq 3$. As the induction hypothesis, we assume that

C: For each $k^{\prime} \in\{1,2, \ldots, k-1\}$, each $\left(a^{\prime}, b^{\prime}, r^{\prime}\right) \in Z\left(R^{0}\right)$, each $\bar{R}^{\prime} \in \underline{\mathcal{R}}\left(a^{\prime}, r^{\prime}\right)$, each $c^{\prime} \in C\left(R^{0},\left(a^{\prime}, r^{\prime}\right), \bar{R}^{\prime}\right)$, and each $\hat{N} \subset N$ with $|\hat{N}|=k^{\prime}, f\left(R_{\hat{N}}^{\left(c^{\prime}\right)}, R_{-\hat{N}}^{\left(a^{\prime}\right)}\right)\left(\left\{c^{\prime}\right\}\right)=0$.

We show that $f\left(R_{N^{\prime}}^{(c)}, R_{-N^{\prime}}^{(a)}\right)(\{c\})=0$. By contradiction, suppose that

$$
f\left(R_{N^{\prime}}^{(c)}, R_{-N^{\prime}}^{(a)}\right)(\{c\})>0 .
$$

Let $j \in N^{\prime}$. Without loss of generality, let $j \equiv 1$. We establish two steps to derive a contradiction.

Step 1 of Claim 1. For each $h \in\{0,1, \ldots, k-1\}$, and each $N_{h} \subseteq N^{\prime} \backslash\{1\}$ such that $\left|N_{h}\right|=h, f\left(\bar{R}_{1}, R_{N_{h}}^{0}, R_{-\left(\{1\} \cup N_{h}\right)}^{(a)}\right)(\{a\})=1$.

We prove Step 1 by induction on $h$. If $h=0$, by Lemma $4, f\left(\bar{R}_{1}, R_{-1}^{(a)}\right)(\{a\})=1$. 
Next, let $h \in\{1, \ldots, k-1\}$ and $N_{h} \subseteq N^{\prime} \backslash\{1\}$ be such that $\left|N_{h}\right|=h$. Without loss of generality, let $N_{h} \equiv\{2,3, \ldots, h+1\}$. As the induction hypothesis, we assume that

D: For each $\bar{N} \subset N^{\prime} \backslash\{1\}$ with $|\bar{N}|=h-1, f\left(\bar{R}_{1}, R_{\bar{N}}^{0}, R_{-(\{1\} \cup \bar{N})}^{(a)}\right)(\{a\})=1$.

Let $i \in N_{h}$ and $\bar{N} \equiv N_{h} \backslash\{i\}$. Without loss of generality, let $i \equiv 2$. Let $\hat{R}_{-N_{h}} \equiv$ $\left(\bar{R}_{1}, R_{-(\{1,2\} \cup \bar{N})}^{(a)}\right)$. By contradiction, suppose that $f\left(R_{2}^{0}, R_{\bar{N}}^{0}, \hat{R}_{-N_{h}}\right)(\{a\})<1$. There are three cases.

Case 1. $f\left(R_{2}^{0}, R_{\bar{N}}^{0}, \hat{R}_{-N_{h}}\right)(] a, r[)>0$.

By induction hypothesis $(\mathrm{D}), f\left(R_{2}^{(a)}, R_{\bar{N}}^{0}, \hat{R}_{-N_{h}}\right)(\{a\})=1$. Then,

$$
\begin{aligned}
f\left(R_{2}^{0}, R_{\bar{N}}^{0}, \hat{R}_{-N_{h}}\right)\left(U\left(R_{2}^{0}, a\right)\right) & \geq f\left(R_{2}^{(a)}, R_{\bar{N}}^{0}, \hat{R}_{-N_{h}}\right)\left(U\left(R_{2}^{0}, a\right)\right) \text { by } s d-S P \\
& =1 \text { by }\{a\} \subset U\left(R_{2}^{0}, a\right) .
\end{aligned}
$$

Thus, $f\left(R_{2}^{0}, R_{\bar{N}}^{0}, \hat{R}_{-N_{h}}\right)(] a, r[)=0$, which is a contradiction.

Case 2. $f\left(R_{2}^{0}, R_{\bar{N}}^{0}, \hat{R}_{-N_{h}}\right)([r, \bar{z}])>0$.

By Lemma $3{ }^{41}$ there is $\left(a^{\prime}, b^{\prime}, r^{\prime}\right) \in Z\left(R^{0}\right)$, and for each $\varepsilon>0$, there is $c^{\prime} \in$ ]$r^{\prime}, r^{\prime}+\varepsilon\left[\right.$ such that $a \leq a^{\prime}<b^{\prime}<r^{\prime}<c^{\prime} \leq r$, and for each $x^{\prime} \in\left[a^{\prime}, c^{\prime}\left[, c^{\prime} P^{0} x^{\prime}\right.\right.$. Let $\bar{R}^{\prime} \in \underline{\mathcal{R}}\left(a^{\prime}, r^{\prime}\right)$ and $c^{\prime} \in C\left(R^{0},\left(a^{\prime}, r^{\prime}\right), \bar{R}^{\prime}\right)$ be such that $c^{\prime} \leq r$. Then, by induction hypothesis (C), $f\left(R_{N_{h}}^{\left(c^{\prime}\right)}, R_{-N_{h}}^{\left(a^{\prime}\right)}\right)\left(\left\{c^{\prime}\right\}\right)=0$.

Since $f\left(R_{2}^{0}, R_{\bar{N}}^{0}, \hat{R}_{-N_{h}}\right)([r, \bar{z}])>0$, by Lemma $5, f\left(R_{N_{h}}^{(\bar{z})}, \hat{R}_{-N_{h}}\right)\left(U\left(R^{(\bar{z})}, r\right)\right)>0$. Thus, by $[r, \bar{z}]=U\left(R^{(\bar{z})}, r\right), f\left(R_{N_{h}}^{(\bar{z})}, \hat{R}_{-N_{h}}\right)([r, \bar{z}])>0$. Since $\left.f\right|_{\mathcal{R}}$ is uncompromising, by $a \leq a^{\prime}<r$ and $p\left(\bar{R}_{1}\right)<r$, we have

$$
f\left(R_{N_{h}}^{(\bar{z})}, R_{-N_{h}}^{\left(a^{\prime}\right)}\right)([r, \bar{z}])=f\left(R_{N_{h}}^{(\bar{z})}, \hat{R}_{-N_{h}}\right)([r, \bar{z}])>0 .
$$

By $[r, \bar{z}] \subseteq\left[c^{\prime}, \bar{z}\right], f\left(R_{N_{h}}^{(\bar{z})}, R_{-N_{h}}^{\left(a^{\prime}\right)}\right)\left(\left[c^{\prime}, \bar{z}\right]\right)>0$.

However, since $\left.f\right|_{\mathcal{R}}=g m^{D}$,

$$
\begin{aligned}
f\left(R_{N_{h}}^{(\bar{z})}, R_{-N_{h}}^{\left(a^{\prime}\right)}\right)\left(\left[c^{\prime}, \bar{z}\right]\right) & =D_{-N_{h}}\left(\left[c^{\prime}, \bar{z}\right]\right) \\
& =1-D_{-N_{h}}\left(\left[\underline{z}, c^{\prime}[)\right.\right. \\
& =D_{N}\left(\left[\underline{z}, c^{\prime}\right]\right)-D_{-N_{h}}\left(\left[\underline{z}, c^{\prime}[) \text { by } D_{N}(\{\underline{z}\})=1\right.\right. \\
& =f\left(R_{N_{h}}^{\left(c^{\prime}\right)}, R_{-N_{h}}^{\left(a^{\prime}\right)}\right)\left(\left\{c^{\prime}\right\}\right) \\
& =0,
\end{aligned}
$$

which is a contradiction.

Case 3. $f\left(R_{2}^{0}, R_{\bar{N}}^{0}, \hat{R}_{-N_{h}}\right)([\underline{z}, a[)>0$.

By Lemma 3, there is $\left(a^{\prime}, b^{\prime}, r^{\prime}\right) \in Z\left(R^{0}\right)$, and for each $\varepsilon>0$, there is $c^{\prime} \in$ ]$r^{\prime}-\varepsilon, r^{\prime}\left[\right.$ such that $a \leq c^{\prime}<r^{\prime}<b^{\prime}<a^{\prime} \leq r$, and for each $\left.\left.x^{\prime} \in\right] c^{\prime}, a^{\prime}\right], c^{\prime} P^{0} x^{\prime}$. Let

\footnotetext{
${ }^{41}$ Note that, since each preference relation $R^{0}$ in $\mathcal{P}$ is continuous, there is a continuous function $g$ on $Z$ that represents $R^{0}$.
} 
$\bar{R}^{\prime} \in \underline{\mathcal{R}}\left(a^{\prime}, r^{\prime}\right)$ and $c^{\prime} \in C\left(R^{0},\left(a^{\prime}, r^{\prime}\right), \bar{R}^{\prime}\right)$ be such that $a \leq c^{\prime}$. Then, by induction hypothesis (C), $f\left(R_{N_{h}}^{\left(c^{\prime}\right)}, R_{-N_{h}}^{\left(a^{\prime}\right)}\right)\left(\left\{c^{\prime}\right\}\right)=0$.

Since $f\left(R_{2}^{0}, R_{\bar{N}}^{0}, \hat{R}_{-N_{h}}\right)\left(\left[\underline{z}, a[)>0\right.\right.$, by Lemma $5, f\left(R_{N_{h}}^{(\underline{z})}, \hat{R}_{-N_{h}}\right)\left(S U\left(R^{(\underline{z})}, a\right)\right)>0$. By $\left[\underline{z}, a\left[=S U\left(R^{(\underline{z})}, a\right), f\left(R_{N_{h}}^{(\underline{z})}, \hat{R}_{-N_{h}}\right)\left(\left[\underline{z}, a[)>0\right.\right.\right.\right.$. Since $\left.f\right|_{\underline{\mathcal{R}}}$ is uncompromising, by $a<p\left(\bar{R}_{1}\right)$ and $a<a^{\prime}$, we have

$$
f\left(R_{N_{h}}^{(\underline{z})}, R_{-N_{h}}^{\left(a^{\prime}\right)}\right)\left(\left[\underline{z}, a[)=f\left(R_{N_{h}}^{(\underline{z})}, \hat{R}_{-N_{h}}\right)([\underline{z}, a[)>0 .\right.\right.
$$

However, since $\left.f\right|_{\underline{\mathcal{R}}}=g m^{D}$ and $a \leq c^{\prime}$, we have

$$
\begin{aligned}
f\left(R_{N_{h}}^{(\underline{z})}, R_{-N_{h}}^{\left(a^{\prime}\right)}\right)([\underline{z}, a[) & =D_{N_{h}}([\underline{z}, a[) \\
& \leq D_{N_{h}}\left(\left[\underline{z}, c^{\prime}\right]\right) \\
& =D_{N_{h}}\left(\left[\underline{z}, c^{\prime}\right]\right)-D_{\emptyset}\left(\left[\underline{z}, c^{\prime}[) \text { by } D_{\emptyset}(\{\bar{z}\})=1\right.\right. \\
& =f\left(R_{N_{h}}^{\left(c^{\prime}\right)}, R_{-N_{h}}^{\left(a^{\prime}\right)}\right)\left(\left\{c^{\prime}\right\}\right) \\
& =0,
\end{aligned}
$$

which is a contradiction.

Since we derive a contradiction for each of the above three cases, we conclude that $f\left(R_{2}^{0}, R_{\bar{N}}^{0}, \hat{R}_{-N_{h}}\right)(\{a\})=1$. Thus, Step 1 of Claim 1 holds.

Step 2 of Claim 1. For each $h \in\{0,1, \ldots, k-1\}$, and each $N_{h} \subseteq N^{\prime} \backslash\{1\}$ such that $\left|N_{h}\right|=h, f\left(R_{1}^{(c)}, R_{N_{h}}^{0}, R_{N^{\prime} \backslash\left(\{1\} \cup N_{h}\right)}^{(c)}, R_{-N^{\prime}}^{(a)}\right)(\{c\})>0$.

We show Step 2 by induction on $h$. If $h=0$, the desired conclusion follows from the inequality (10).

Next, let $h \in\{1, \ldots, k-1\}$ and $N_{h} \subseteq N^{\prime} \backslash\{1\}$ be such that $\left|N_{h}\right|=h$. Without loss of generality, let $N_{h} \equiv\{2,3, \ldots, h+1\}$. As the induction hypothesis, we assume that

E: For each $\bar{N} \subset N^{\prime} \backslash\{1\}$ with $|\bar{N}|=h-1, f\left(R_{1}^{(c)}, R_{\bar{N}}^{0}, R_{N^{\prime} \backslash(\{1\} \cup \bar{N})}^{(c)}, R_{-N^{\prime}}^{(a)}\right)(\{c\})>0$.

Let $i \in N_{h}$ and $\bar{N} \equiv N_{h} \backslash\{i\}$. Without loss of generality, let $i \equiv 2$. Let $\tilde{R}_{-N_{h}} \equiv\left(R_{1}^{(c)}, R_{N^{\prime} \backslash(\{1,2\} \cup \bar{N})}^{(c)}, R_{-N^{\prime}}^{(a)}\right.$. We show that $f\left(R_{2}^{0}, R_{\bar{N}}^{0}, \tilde{R}_{-N_{h}}\right)(\{c\})>0$. First, by induction hypothesis (E), $f\left(R_{2}^{(c)}, R_{\bar{N}}^{0}, \tilde{R}_{-N_{h}}\right)(\{c\})>0$. Then,

$$
\begin{aligned}
f\left(R_{2}^{0}, R_{\bar{N}}^{0}, \tilde{R}_{-N_{h}}\right)\left(U\left(R_{2}^{0}, c\right)\right) & \geq f\left(R_{2}^{(c)}, R_{\bar{N}}^{0}, \tilde{R}_{-N_{h}}\right)\left(U\left(R_{2}^{0}, c\right)\right) \text { by } s d-S P \\
& >0 \text { by }\{c\} \subset U\left(R_{2}^{0}, c\right) .
\end{aligned}
$$

Thus, $f\left(R_{2}^{0}, R_{\bar{N}}^{0}, \tilde{R}_{-N_{h}}\right)([\underline{z}, a[\cup[c, \bar{z}])>0$. By contradiction, suppose that $f\left(R_{2}^{0}, R_{\bar{N}}^{0}, \tilde{R}_{-N_{h}}\right)(\{c\})=0$. There are two cases.

Case 1. $\left.\left.f\left(R_{2}^{0}, R_{\bar{N}}^{0}, \tilde{R}_{-N_{h}}\right)(] c, \bar{z}\right]\right)>0$.

By Lemma $5, f\left(R_{N_{h}}^{(\bar{z})}, \tilde{R}_{-N_{h}}\right)\left(S U\left(R^{(\bar{z})}, c\right)\right)>0$. Thus, $\left.\left.f\left(R_{N_{h}}^{(\bar{z})}, \tilde{R}_{-N_{h}}\right)(] c, \bar{z}\right]\right)>0$. 
However, by induction hypothesis $(\mathrm{C}), f\left(R_{N_{h}}^{(c)}, R_{-N_{h}}^{(a)}\right)(\{c\})=0$. Since $\left.f\right|_{\underline{\mathcal{R}}}=$ $g m^{D}$ and $N_{h}=\{2\} \cup \bar{N}$,

$$
\begin{aligned}
\left.\left.f\left(R_{N_{h}}^{(\bar{z})}, \tilde{R}_{-N_{h}}\right)(] c, \bar{z}\right]\right) & \left.\left.=D_{-N_{h}}(] c, \bar{z}\right]\right) \\
& \leq D_{-N_{h}}([c, \bar{z}]) \\
& =1-D_{-N_{h}}([\underline{z}, c[) \\
& =D_{N}([\underline{z}, c])-D_{-N_{h}}\left(\left[\underline{z}, c[) \text { by } D_{N}(\{\underline{z}\})=1\right.\right. \\
& =f\left(R_{N_{h}}^{(c)}, R_{-N_{h}}^{(a)}\right)(\{c\}) \\
& =0
\end{aligned}
$$

which is a contradiction.

Case 2. $f\left(R_{2}^{0}, R_{\bar{N}}^{0}, \tilde{R}_{-N_{h}}\right)([\underline{z}, a[)>0$.

By Lemma 3, there is $\left(a^{\prime}, b^{\prime}, r^{\prime}\right) \in Z\left(R^{0}\right)$, and for each $\varepsilon>0$, there is $c^{\prime} \in$ ]$r^{\prime}-\varepsilon, r^{\prime}\left[\right.$ such that $a \leq c^{\prime}<r^{\prime}<b^{\prime}<a^{\prime} \leq r$, and for each $\left.\left.x^{\prime} \in\right] c^{\prime}, a^{\prime}\right], c^{\prime} P^{0} x^{\prime}$. Let $\bar{R}^{\prime} \in \underline{\mathcal{R}}\left(a^{\prime}, r^{\prime}\right)$, and $c^{\prime} \in C\left(R^{0},\left(a^{\prime}, r^{\prime}\right), \bar{R}^{\prime}\right)$ be such that $a \leq c^{\prime}$. Then, by induction hypothesis $(\mathrm{C}), f\left(R_{N_{h}}^{\left(c^{\prime}\right)}, R_{-N_{h}}^{\left(a^{\prime}\right)}\right)\left(\left\{c^{\prime}\right\}\right)=0$.

Since $f\left(R_{2}^{0}, R_{\bar{N}}^{0}, \tilde{R}_{-N_{h}}\right)\left(\left[\underline{z}, a[)>0\right.\right.$, by Lemma $5, f\left(R_{N_{h}}^{(\underline{z})}, \tilde{R}_{-N_{h}}\right)\left(S U\left(R^{(\underline{z})}, a\right)\right)>0$. Thus, $f\left(R_{N_{h}}^{(\underline{z})}, \tilde{R}_{-N_{h}}\right)\left(\left[\underline{z}, a[)>0\right.\right.$. Since $\left.f\right|_{\underline{\mathcal{R}}}$ is uncompromising, by $a<a^{\prime}<c$,

$$
f\left(R_{N_{h}}^{(\underline{z})}, R_{-N_{h}}^{\left(a^{\prime}\right)}\right)\left(\left[\underline{z}, a[)=f\left(R_{N_{h}}^{(\underline{z})}, \tilde{R}_{-N_{h}}\right)([\underline{z}, a[)>0 .\right.\right.
$$

However, since $\left.f\right|_{\underline{\mathcal{R}}}=g m^{D}, a \leq c^{\prime}$, and $N_{h}=\{2\} \cup \bar{N}$,

$$
\begin{aligned}
f\left(R_{N_{h}}^{(\underline{z})}, R_{-N_{h}}^{\left(a^{\prime}\right)}\right)([\underline{z}, a[) & =D_{N_{h}}([\underline{z}, a[) \\
& \leq D_{N_{h}}\left(\left[\underline{z}, c^{\prime}\right]\right) \\
& =D_{N_{h}}\left(\left[\underline{z}, c^{\prime}\right]\right)-D_{\emptyset}\left(\left[\underline{z}, c^{\prime}[) \text { by } D_{\emptyset}(\{\bar{z}\})=1\right.\right. \\
& =f\left(R_{N_{h}}^{\left(c^{\prime}\right)}, R_{-N_{h}}^{\left(a^{\prime}\right)}\right)\left(\left\{c^{\prime}\right\}\right) \\
& =0,
\end{aligned}
$$

which is a contradiction.

Since we derive a contradiction for each of the above two cases, we conclude that $f\left(R_{2}^{0}, R_{\bar{N}}^{0}, \tilde{R}_{-N_{h}}\right)(\{c\})>0$, and so, Step 2 of Claim 1 holds.

Next, we complete the proof of Claim 1. By Step 1 of Claim 1 for $N_{h} \equiv N^{\prime} \backslash\{1\}$, $f\left(\bar{R}_{1}, R_{N^{\prime} \backslash\{1\}}^{0}, R_{-N^{\prime}}^{(a)}\right)(\{a\})=1$. Since $c \bar{P}_{1} a, f\left(\bar{R}_{1}, R_{N^{\prime} \backslash\{1\}}^{0}, R_{-N^{\prime}}^{(a)}\right)\left(U\left(\bar{R}_{1}, c\right)\right)=0$. By Step 2 of Claim 1 for $N_{h} \equiv N^{\prime} \backslash\{1\}, f\left(R_{1}^{(c)}, R_{N^{\prime} \backslash\{1\}}^{0}, R_{-N^{\prime}}^{(a)}\right)(\{c\})>0$. Thus,

$$
f\left(R_{1}^{(c)}, R_{N^{\prime} \backslash\{1\}}^{0}, R_{-N^{\prime}}^{(a)}\right)\left(U\left(\bar{R}_{1}, c\right)\right)>0=f\left(\bar{R}_{1}, R_{N^{\prime} \backslash\{1\}}^{0}, R_{\left.-N^{\prime}\right)}^{(a)}\right)\left(U\left(\bar{R}_{1}, c\right)\right),
$$

which contradicts sd-strategy-proofness.

Finally, we complete the proof of Theorem 2. By Lemma 3, there is $(a, b, r) \in$ $Z\left(R^{0}\right)$, and for each $\varepsilon>0$, there is $\left.c \in\right] r, r+\varepsilon[$ such that $x \leq a<b<r<c \leq z$, and for each $x^{\prime} \in\left[a, c\left[, c P^{0} x^{\prime}\right.\right.$. Let $R^{d} \in \underline{\mathcal{R}}(a, r)$ and $c \in C\left(R^{0},(a, r), R^{d}\right)$. Then, by Claim 1 for $N^{\prime} \equiv N, f\left(R_{N}^{(c)}\right)(\{c\})=0$. However, since $\left.f\right|_{\mathcal{R}}=g m^{D}$ and $g m^{D}$ is unanimous, $f\left(R_{N}^{(c)}\right)(\{c\})=1$, which is a contradiction. 


\section{References}

Barberà, S. (1979a) A note on group strategy-proof decision schemes. Econometrica 47: 637-640

Barberà, S. (1979b) Majority and positional voting in a probabilistic framework. Review of Economic Studies 46: 379-389

Barberà, S., Massó, J., and Neme, A. (1999) Maximal domains of preferences preserving strategy-proofness for generalized median voter schemes. Social Choice and Welfare 16: 321-336

Barberà, S., Sonnenschein, H., and Zhou, L. (1991) Voting by committees. Econometrica 59: 595-609

Berga, D. (2002) Single-peakedness and strategy-proofness of generalized median voter schemes. Social Choice and Welfare 19: 175-192

Berga, D. and Serizawa, S. (2000) Maximal domain for strategy-proof rules with one public good. Journal of Economic Theory 90: 39-61

Border, K.C. and Jordan, J.S. (1983) Straightforward elections, unanimity and phantom voters. Review of Economic Studies 50: 153-170

Ching, S. (1997) Strategy-proofness and "median voters". International Journal of Game Theory 26: 473-490

Ehlers, L. and Klaus, B., (2001) Solidarity and probabilistic target rules. Journal of Public Economic Theory 3: 167-184

Ehlers, L., Peters, H., and Storcken, T. (2002) Strategy-proof probabilistic decision schemes for one-dimensional single-peaked preferences. Journal of Economic Theory 105: 408-434

Dutta, B., Peters, H., and Sen, A. (2002) Strategy-proof probabilistic mechanisms in economies with pure public goods. Journal of Economic Theory 106: 392416

Gibbard, A. (1977) Manipulation of schemes that mix voting with chance. Econometrica 45: 665-681

Hatsumi, K., Berga, D., and Serizawa, S. (2011) A maximal domain for strategyproof and no-vetoer rules in the multi-object choice model. Osaka University, ISER Discussion Paper No. 809

Massó, J. and Moreno de Barreda, I. (2011) On strategy-proofness and symmetric single-peakedness. Games and Economic Behavior 72: 467-484

Mizobuchi, H. and Serizawa, S. (2006) Maximal domain for strategy-proof rules in allotment economies. Social Choice and Welfare 27: 195-210 
Moulin, H. (1980) On strategy-proofness and single-peakedness. Public Choice 35: 437-456

Serizawa, S. (1995) Powers of voters and domain of preferences where voting by committees is strategy-proof. Journal of Economic Theory 67: 599-608

Thomson, W. (1999) Phantom voters and calibration points; a terminological note. Working paper, University of Rochester.

Thomson, W. (2010) Strategy-proof Resource Allocation Rules. Mimeo. 